\title{
Layout optimisation for an installation port of an offshore wind farm
}

\author{
Chandra Ade Irawan ${ }^{1, *}$, Xiang Song ${ }^{1}$, Dylan Jones ${ }^{1}$, Negar Akbari ${ }^{2}$ \\ ${ }^{1}$ Centre for Operational Research and Logistics, Department of Mathematics, University of Portsmouth, \\ Lion Gate Building, Lion Terrace, Portsmouth, PO1 3HF, UK \\ ${ }^{2}$ The Logistics Institute, University of Hull, Hull, UK, HU6 7RX
}

\begin{abstract}
This paper investigates a port layout problem, where the layout of an installation port for an offshore wind farm needs to be generated in an efficient way so as to minimise the transportation cost of main components of an offshore wind turbine within the port. Two mixed integer linear programming (MILP) models are established to configure the optimal port layout, where the shapes of subareas that need to be located in the port are rectangular with several possible dimensional configurations to select from and the shape of the port area can be treated as either a convex or a concave polygon. The MILPs can be solved to optimality for small-sized problems. Matheuristic approaches based on Variable Neighbourhood Search (VNS) and an exact method (MILP) are also proposed to find solutions for medium-sized problems. The methods are assessed using randomly generated data sets. In addition, the area of a proposed Scottish port is used as a case study. The results obtained from the computational experiments validate the effectiveness of the proposed matheuristic approaches.
\end{abstract}

Key words: Layout optimisation, offshore wind farm, matheuristic approach, VNS, MILP.

\section{Introduction}

Within two decades of the installation of the first offshore wind farm (OWF) in 1991, the offshore wind industry has experienced a substantial growth in terms of the number of projects and the capacity per project. Although in the late 1990s single wind turbines (WT) with power ratings less than hundreds of KW were installed, today offshore wind farms are planned with capacities above $1 \mathrm{GW}$. Thus, it is fair to say that OWF have generation capacities comparable to that of existing conventional power plants (Perveen et al. 2014). By the end of 2014, there were $25 \mathrm{GW}$ of consented offshore wind projects in Europe (EWEA,

\footnotetext{
* Corresponding author.

E-mail address: chandra.irawan@ port.ac.uk
} 
2015); this remarkable growth in the industry is matched by its significant future potential and also reflects the need of developing ports and onshore infrastructure to effectively support the planned OWFs.

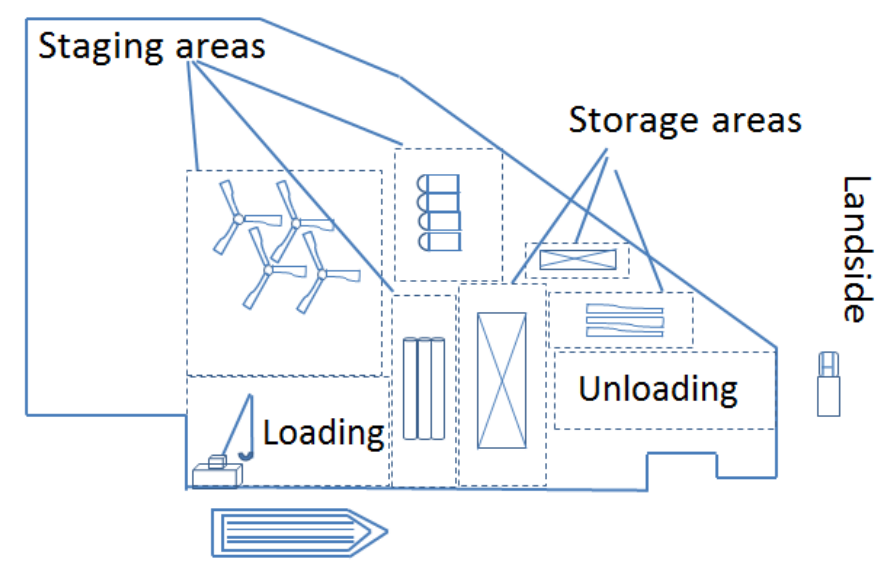

Seaside

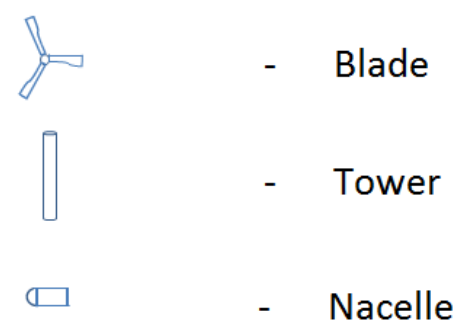

Figure 1 Schematic top view of an offshore wind farm port layout

For the development of this industry, ports play a fundamental role and its rapid growth imposes significant requirements on the ports and their characteristics. The efficient installation of an offshore wind farm depends on the proper setup in the port area. If the port's layout and access lanes are ideal, the turnaround time for an installation cycle will not be unnecessarily lengthened. Also efficient layout configuration can result in a significant reduction in the initial investment and in the resultant long term operational costs (Tompkins et al., 1996). The opposite however, will put constraints on all parts of the project (Thomson, 2012).

The wind farm port layout problem has the similarity to the facility layout problem (FLP) and container port layout problems. The port area can be segmented into subareas: unloading areas, storage areas, staging areas and loading areas (see Figure 1). The components (including nacelle, tower and blades) are unloaded at the unloading area along the road of the entrance of the offshore wind port where each component is stored at its respective storage area. The components are then taken to the staging area allocated to each component where further preparation and assembly is performed. Lastly, components are taken to the loading area at the quayside where they will be loaded on the heavy lift vessels and taken offshore. It is recognised that the average container port item is lighter, smaller and more regularly shaped than a wind farm component. The heavy and irregular shaped wind farm components require a platform vehicle with a large array of wheels to transport, which 
is called Self-propelled modular transport vehicles (SPMT). The unit transportation cost of the SPMT is relatively very expensive. The layout of a port servicing the offshore wind industry should hence be optimised in order to minimise the total transportation cost.

The facility layout problem (FLP) is the placement of the facilities with known dimensions in the plant area to minimize operating cost and maximize system efficiency. FLP exists in various contexts in the literature, e.g., positioning machines in automated manufacturing systems or locating buildings on a factory premises. A FLP generally has a set of constraints as follows: (1) all facilities must be located within a given plant area; (2) these facilities must not overlap with one another, and some facilities must be fixed at certain locations or forbidden for being in specific regions (Meller \& Gau, 1996). Recent publications on FLP addressed more complicated and realistic constraints, like: (3) the layout must fulfil aspect ratio (height to width or width to height) constraints for the dimension of facilities, because facilities with proper aspect ratios are more practical in real-world applications (Tam and Li, 1991; Chwif et al., 1998). More detailed definition about aspect ratio will be illustrated later; (4) Facilities or the plant are of complicated geometric shapes (Lee and Kim, 2000, Chen et al., 2015); (5) A limited number of models in FLP can accommodate the structure of aisles (Zhang et al., 2009). (6) The layout's efficiency is measured in terms of the closeness rating of the two facilities (Neghabi and Tari, 2016). A more detailed review on FLPs will be provided in Section 2. A solution to the FLP is a layout that specifies the relative location and dimensions of each facility.

In the offshore wind farm port layout problem, both general and specific constraints (1) to (5) in FLP are considered. As shown in Figure 1, each subarea can be represented as a rectangular block, considering its area, orientation and aspect ratio (Tam and $\mathrm{Li}, 1991)$. The two orientations considered for each subarea are horizontal or vertical to the port seaside, and according to the offshore wind farm port layout practice, both orientations are allowed for each subarea. The aspect ratio of the subarea is defined as the ratio of the subarea's long side length to its shorter side length. The areas, minimum (Min) and maximum (Max) aspect ratios, and orientation of the subarea $i$ are specified in Table 1 as an example.

Table 1. The area, aspect ratios and orientation of the subarea $i$

\begin{tabular}{ccccc}
\hline Subarea $\boldsymbol{i}$ & Area & Min Aspect Ratio & Max Aspect Ratio & Orientation \\
\hline Loading Area & $50,000 \mathrm{~m}^{2}$ & 1.12 & 1.25 & Horizontal or Vertical \\
\hline
\end{tabular}


The feature that the port area may need to be represented as an irregular shape based on its geographic characteristics complicates the FLP problem. For example, some of solution techniques dealing with rectangular shaped facility and/or plant area may not be applicable (Heragu and Kusiak, 1991; Meller et al., 1999), e.g., when a plant area has a rectangular shape, the aspect ratio can be used to restrict the occurrence of an extremely long and narrow facility. However, when a plant area has an arbitrary shape, dealing with aspect ratios is challenging. In the literature, only one study is found in the FLP literature, which dealt with both an irregular shaped plant area and aspect ratio (Chen et al., 2015). The slicing structure (Chen et al., 2015) applied to divide the irregular shape logistics park into several nonoverlapping regions is not accurate enough to measure the distance between two subareas in the offshore wind port layout problem considered by this paper.

In this paper, to cope with aspect ratio, aisle and irregular shape of the port area at the same time, the actual lengths and widths of the subarea $i$ are predefined by the offshore wind farm port layout decision maker according to the dimensions of the components, aisle area required by the vehicles, aspect ratios and required rotations. For example, the storage area for each component can accommodate 50 pieces of each component, and the staging area for each component can accommodate 4 pieces of each component. The lengths and widths of the actual areas of the subarea $i$ defined in Table 1 are presented in Table 2 . The aspect ratios are within the ranges defined in Table 1 and the requirements of the orientation of the subareas are satisfied. Different orientations have been considered as different actual sizes of the same subarea. In each rectangle, the aisle area has been included. To our best knowledge, no other papers in FLPs have ever dealt with aspect ratio, aisle and irregular shape at the same time for this type of port layout problem.

Table 2. Lengths $(l)$ and widths ( $w)$ of the actual areas of subarea $i$

\begin{tabular}{clllllllllll}
\hline Subarea $\boldsymbol{i}$ & \multicolumn{4}{c}{ Rectangle 1 } & \multicolumn{2}{l}{ Rectangle 2 } & \multicolumn{2}{l}{ Rectangle 3 } & \multicolumn{2}{c}{ Rectangle 4 } & \multicolumn{2}{c}{ Rectangle 5 } \\
\cline { 2 - 13 } & $l$ & $w$ & $L$ & $w$ & $l$ & $w$ & $l$ & $w$ & $l$ & $w$ \\
Loading Area & 250 & 200 & 200 & 250 & 238 & 210 & 210 & 238 & 246 & 203 \\
\hline
\end{tabular}

Different from the constraint (6) in the FLP, the port layout problem aims to generate a feasible port layout satisfying constraint (1) to (5) for an offshore wind port with the minimum total transportation cost of the components' movements between subareas, where the transportation cost is defined as a linear function of the rectilinear distance between the centres of two rectangles (Chwif et al., 1998). The closeness relationships of the subareas 
have been considered using a binary matrix indicating whether each component will move from one subarea to another (See Section 3.1).

From a geometric point of view, the offshore wind farm port layout problem can be considered as assigning a set of appropriate rectangular areas (e.g. loading, unloading, storage, and stage areas) to a port area of irregular shape, where each rectangle is selected from a cluster of rectangles of different sizes (as shown in Table 2). There are in total $K$ clusters/subareas to consider and the set of appropriate rectangles are composed of one and exactly one rectangle from each cluster. If the $K$ rectangles are chosen from the $K$ clusters before the assignment process starts, the problem can be simplified to $2 \mathrm{D}$ irregular shape Single Bin Size Bin Packing Problem (SBSBPP) according to the typology of cutting and packing problems from Wäscher et al. (2007). We refer to our problem as a generalized 2D irregular shape SBSBPP. To our best knowledge, there is no such paper in the literature of cutting and packing problems available for this port layout problem due to the fact that a choice of a rectangle from each cluster needs to be made during the assignment process. A detailed review on SBSBPP will be provided in Section 2.

Unfortunately, both layout problems and cutting and packing problems are known to be complex and are generally NP-Hard (Garey and Johnson,1979). As a result, the optimal solution can be found only for small and medium sized problems and heuristic algorithms need to be designed to find efficient solutions.

This paper is divided into 6 main sections. In the next section (Section 2), an overview of the literature regarding facility layout problems, port layout problems, and relevant packing and cutting papers is given. Section 3 presents the formulations of the proposed port layout models for an offshore wind port. The description of the proposed matheuristic methods for solving medium-sized problems is given in Section 4. In Section 5, the computational study is presented followed by conclusions and suggestions for future work in Section 6.

\section{Literature Review}

Given the dearth of offshore wind port literature, and the similarity of some of the operations between facility layout problem (FLP), container ports and this offshore wind farm port layout problem, a review of the FLP and container port literature has been 
conducted to assess the methodologies that have been applied to deal with layout optimisation problems. Further, from geometric point of view, this offshore wind farm port layout problem is a SBSBPP. We first review FLP, then the container port layout problem and finally the SBSBPP.

\section{Facility Layout Problem}

Different models of FLP are found in the literature, most of which have been examined in a deterministic state, and a limited number (Balakrishnan et al., 2003; Meng, Heragu, \& Zijm, 2004) have been studied in a dynamic state. In terms of the objective function, most of the presented models have one objective (Kulturel-Konak and Konak, 2011), whereas the rest (Matai, 2015; Ripon et al., 2013; McKendall et al., 1999; Askin and Standridge, 1993, p.204) are multi-objective. In the literature, most of the studies focus on single floor FLPs, whereas recently, multi-floor facility layout received attention as land supply becomes increasingly insufficient and expensive (Lee, Roh, \& Jeong, 2005). For detailed survey of the variants of the FLP, please refer to Drira et al. (2007). This review focuses on mathematical formulations of single objective, single floor and deterministic FLPs and corresponding heuristic solution methods, which is the case of the problem in this paper. Both discrete and continuous formulations of the FLPs have been examined frequently in the literature, which can lead to Quadratic Assignment Problems (QAP) and Mixed Integer Programmings (MIP) respectively.

The QAP was introduced first by Koopmans and Beckman (1957) to solve the equal area facility layout problem under the assumption that all locations are fixed and known a priori. Based on Kusiak and Heragu (1987) the unequal area FLP could be modelled as a modified QAP by breaking the departments into small grids with equal area, assigning a large artificial flow between those grids of the same department to ensure that they are not split. However due to the increase in departments, it is not possible to solve even small problems with a few unequal area departments (Meller and Gau, 1996). Bozer and Meller (1993) show that such approach is ineffective because it implicitly adds a department shape constraint. In addition, the discrete representations of QAP are not suited to represent the exact position of facilities in the plant area and the some specific constraints cannot be expressed appropriately by QAP, e.g. orientation of facilities. 
The continuous representation of the FLPs is often addressed as a Mixed Integer Programming Problem (Das, 1993). Heragu and Kusiak (1991) proposed one of the first MIP formulations of the FLP on the continuous plan. Different from QAP, the location of the sites need not be known a priori. In their model, the length, width and orientation of the departments are predefined in their single row and multiple row FLPs. The model includes disjunctive constraints to prevent facility overlaps. A heuristic algorithm has been provided to solve the model with up to 30 facilities in acceptable running time. Meanwhile, Montreuil (1990) introduced a modelling framework for integrating layout design and flow network design, which was structured in an ever increasing scope and complexity. An MIP model for block layout and I/O station location which minimizes inter-station rectilinear travel is proposed. The model includes both constraints to prevent facility overlaps and bounded perimeter constraints to enforce specified facility area and shape requirements. The largest problem instance solved optimally by Montreuil's original formulation had six facilities. Meller et al. (1999) reformulate Montreuil (1990) model by redefining the binary variables and tightening the department area constraints. They propose some general classes of valid inequalities based on the acyclic subgraph structure of their model. Using these inequalities in a branch and bound algorithm, they are able to moderately increase the range of solvable problems to 8 facilities. Sherali et al. (2003) applied polyhedral outer approximation to the facility area to improve the MIP model motivated by Meller et al. (1999), which is able to produce more accurate solution of up to 9 facilities while decreasing the solution effort time. Castillo and Westerlund (2005) developed an $\varepsilon$-accurate approximation. However, the largest problem solved had only nine facilities. Recent authors (Gonçalves, J. F. \& Mauricio G. C., 2015) provide an overview of MIP models for the FLP and propose a random-key genetic algorithm for the unequal area FLP problem.

It is found that most of the MIPs of the FLPs in the literature examined the aspect ratio in rectangular shaped FLPs. The only study found in the FLP literature, which considered the aspect ratio in an irregular shaped logistics park area (Chen et al., 2015) has applied slicing structure to the problem, which cannot reflect the precise location of the subarea in our OWF port layout problem. The above considerations were partial motivation for this research paper. 


\section{Port Layout}

Two major compartments within a container port have been considered in the literature, which are quay side and yard side (Rashidi and Tsang, 2013). The Container port layout problem, also called the yard layout problem in the literature, is one of the optimisation problems in the yard side (Liu et al., 2004; Kim et al., 2008; Petering, 2008; Wiese et al., 2010). We focus our review on yard layout problems.

Despite the influence of the yard layout problem on the productivity of the container handling operation, this problem has been studied less frequently in the literature. Liu et al. (2004), Petering (2008), and Kim et al. (2008) compare the performance of two different yard layouts: (i) the parallel layout, in which the container blocks are laid out parallel to the berth and (ii) the perpendicular layout, in which the blocks are laid out perpendicular to the direction of the berth. In the first two studies container terminals are simulated. The first study considers the effect of the yard layout on the performance of automated container terminals and suggests that the perpendicular layout is superior. The second study considers different performance measures such as the quay crane rate and the productivity of trucks and suggests that the parallel layout is superior. The third study uses an equation based approach, in which formulae for estimating the expected number of relocations for picking up a container from a given layout of the yard, and the expected travel distance of yard trucks for storages and retrievals in a given layout of a container yard are proposed. They find that the parallel model has a higher performance. Further, Wiese et al. (2010) propose mathematical models to solve the yard layout problem that consists of finding the optimal number of driving lanes and the optimal position of those driving lanes for perpendicular or parallel layout with the objective of minimizing the total cost. They consider both rectangular and non-rectangular port shapes.

Among the four mentioned literature works on container port layout problems, all the container blocks have the same predefined orientation: parallel or perpendicular to the yard. In addition, the straight driving lanes separate the container blocks in a slicing way as described in the slicing structure in the FLPs, which simplify the geometric non-overlapping requirement of the container blocks. Finally, no aspect ratio is needed to define the shape of the container blocks. Our OWF Port Layout problem has more similarities to the continuous representation of the FLPs than the yard layout problems. 


\section{D irregular shape Single Bin Size Bin Packing Problem (SBSBPP)}

Although comprehensive research has been carried out and various approaches have been proposed to solve the 1D SBSBPP and 2D rectangular shape SBSBPP (Dowsland and Dowsland, 1992; Valerio de Carvalho, 1999; Hopper and Turton, 2001; Lodi et al., 2002a, 2002b; Vanderbeck, 1999), the research into the irregular shape SBSBPP is rare and frequently reduces the problem to a $1 \mathrm{D}$ problem using a finite set of predetermined patterns (Degraeve and Vandebroek, 1998; Martens, 2004). Only two recent publications are found dealing with irregular shape SBSBPP directly (Han et al. 2013, Martinez-Sykora et al, 2015).

One distinct problem arising from packing rectangular shapes to an irregular stock sheet is the complexity of evaluating the feasibility of an assignment, i.e. do the rectangles overlap, and are they contained inside the irregular port. These geometric issues are an inherent part of irregular packing problems and as a result, fast and accurate evaluation of the geometry is an essential feature when developing solution approaches to these problems. In recent years, Stoyan and Romanova (Stoyan et al., 2001, 2004; Scheithauer et al., 2003, 2005; Chernov et al., 2009) propose a new methodology called Phi-functions for evaluating the geometry in both two-dimensional and three-dimensional irregular packing problems. A phi-function provides a complete mathematical expression to describe the interaction between two geometric objects called phi-objects. Specifically, given the translational position and orientation of each object as the input to the function, it will return the value zero if the two objects touch, a positive number if they are separated, and a negative number if they have common interior points. The value of the number is an indication of the distance between the objects and when the function is normalized will be the minimum Euclidean distance.

In this paper, the Phi-function of rectangles will be adopted to untangle the nonoverlapping among the rectangular shapes (Alvarez-Valdes et al., 2013; Chernov et al., 2009), and the basic geometric knowledge, which can be used to judge if a point is on the right hand side of a line, is used to judge if all the rectangular shapes are contained inside the port of convex shape. A port of concave shape is also considered by inserting dummy rectangles and forbidden convex polygons to forbid certain areas inside the convex polygon from being used. 


\section{Formulation of port layout models for an OWF}

The description of the port layout models for an OWF has been provided in Section 1. The main factors considered in the model are depicted in Figure 2 where the main inputs required by the model include the set of edges that make up a polygon (the port area), set of subareas, set of possible rectangles for each subarea, set of components, set of predefined subareas, and the unit transportation cost of each component (£/metre). A subset of the subareas need to be allocated in a way that at least one corner point of the corresponding rectangle touches a predefined subset of edges of the polygon, which represents the port area. This subset of the subareas is called the set of predefined subareas throughout this paper. The requirement of the predefined subarea is realistic. For example, the loading area needs to be located in the quay side (the boundary of the port area) while the unloading area should be near to the main road (the boundary of the port area). A rectangle representing a predefined subarea may touch more than one edge of the polygon (port area).
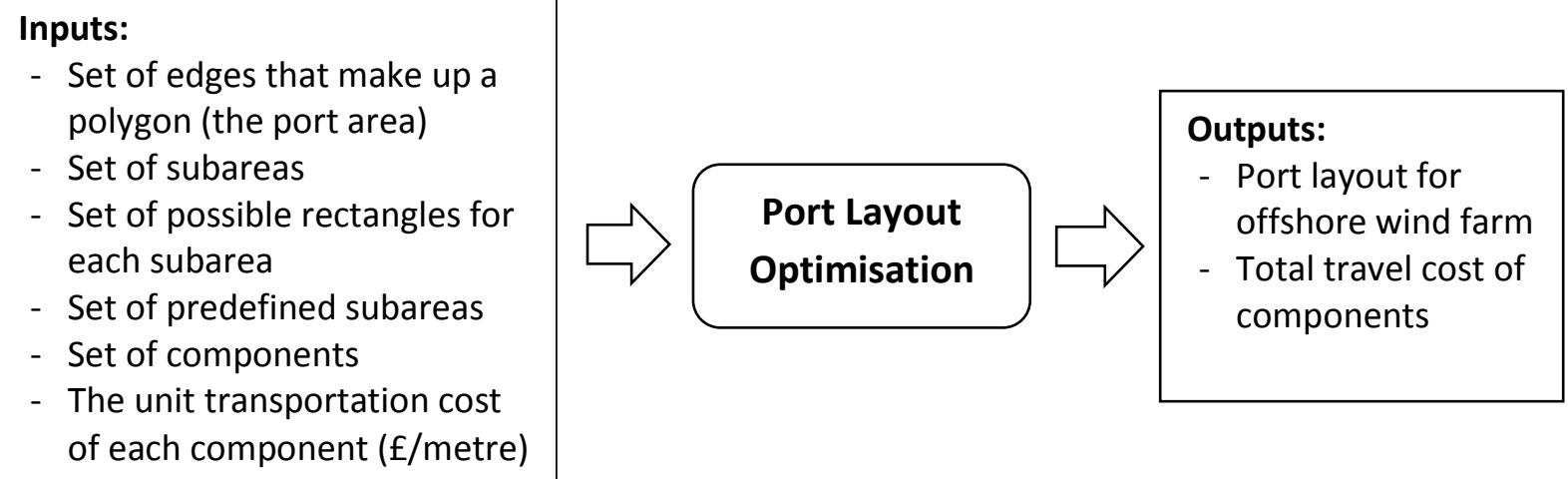

Figure 2. The proposed model

As described in the introduction, the shapes of the subareas are approximated by rectangles with the lengths and the widths of the rectangles parallel to the predefined $\mathrm{x}$ - and $y$-axis respectively. For each subarea, a cluster of rectangles are available to approximate the subarea. The movement of the components from one subarea to another is very expensive as the offshore wind turbine is made up from heavy components such as tower, blades, and nacelle. Thus the total transportation cost to be minimised in this port layout problem is decided by the rectilinear distance between the centres of two rectangles representing two subareas, the known parameters on unit transportation cost (£/metre) for each component and known parameters on whether each component will move from one subarea to another. 
We propose two variant Mixed Integer Linear Programming (MILP) models of the port layout problem for an offshore wind farm. The first model deals with the problem with a port area of convex shape whereas the second one addresses the problem with a concave polygon. We refer to the first model as the Basic MILP (BMILP) model whereas the second one is called the Enhanced MILP (EMILP), which is an enhancement of the BMILP model.

\subsection{The Basic MILP (BMILP) model}

This subsection presents the mathematical formulation of the Basic MILP (BMILP) model where each subarea has a set of possible rectangles to choose from and the shape of the port area is assumed to be a convex polygon. As all subareas (represented by rectangles) need to be inside the port area (represented by a convex polygon), all the corner points of each rectangle must be inside the convex polygon. To realize this constraint, the vertices of the convex polygon are ordered clockwise first (see Figure 3a). A point is on the interior of this convex polygon if it is always on the right hand side of all the line segments. To judge if a point is on the right hand side of each edge of the convex polygon, the following basic geometrical knowledge is provided first.

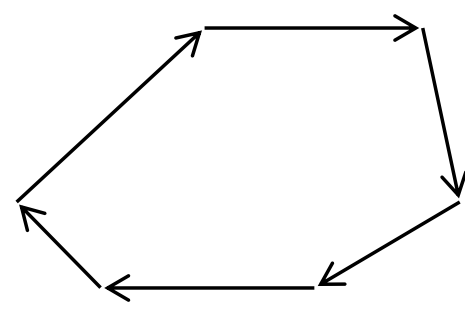

(a)

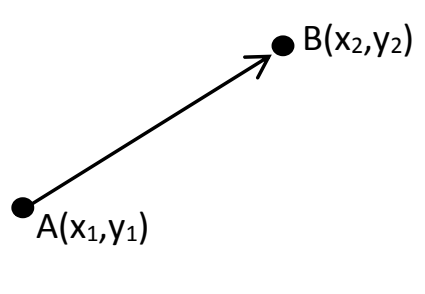

(b)

Figure 3. The formulation of the parameters of the edges

Let $E$ be a set of edge vectors indexed by $e$ with $\alpha_{e}, \beta_{e}$, and $\gamma_{e}$ as the parameters of edge $e(e \in E)$. The formulation to calculate the value of the parameters is given in Figure $3 \mathrm{~b}$ where edge $e$ with the direction from point $\mathrm{A}$ to point $\mathrm{B}$ is given. A point, say point $\mathrm{C}$ $\left(x_{c}, y_{c}\right)$ will be on the right hand side of the edge $e$, if $\alpha_{e} \cdot x_{c}+\beta_{e} \cdot y_{c}+\gamma_{e}<0$, and point $\mathrm{C}$ lies on edge $e$, if $\alpha_{e} \cdot x_{c}+\beta_{e} \cdot y_{c}+\gamma_{e}=0$. In the case that the $\alpha_{e} \cdot x_{c}+\beta_{e} \cdot y_{c}+\gamma_{e}>0$, point $\mathrm{C}$ is on the left hand side of edge $e$. It means that point $\mathrm{C}$ will be inside or on the edge of the convex polygon if $\alpha_{e} \cdot x_{c}+\beta_{e} \cdot y_{c}+\gamma_{e} \leq 0, \forall e \in E$. The following notations are used to describe the sets and parameters of the BMILP model. 


\section{$\underline{\text { Sets and index }}$}

$A \quad=$ set of subareas with $a$ as its index where $K=|A|$.

$Q \quad=$ set of components with $q$ as its index.

$E \quad=$ set of edges direction with $e$ as its index. The edges make up a polygon which is the area of the port.

$\hat{A} \quad=$ set of predefined subareas (represented by rectangles) whose at least one corner point lies on the border of polygon, $\hat{A} \subset A$.

$E_{a} \quad=$ set of edges that at least one corner point of the rectangle representing subarea $a$ $(a \in \hat{A})$ lies on these edges, $E_{a} \subset E$.

$S_{a} \quad=$ set of possible rectangles for subarea $a(a \in A)$ with $s$ as its index.

\section{$\underline{\text { Parameters }}$}

$n \quad=$ total number of subareas $(n=|A|)$

$w_{a, s}=$ the width of rectangle $s$ representing subarea $a\left(a \in A, s \in S_{a}\right)$

$l_{a, s} \quad=$ the length of rectangle $s$ representing subarea $a\left(a \in A, s \in S_{a}\right)$

$c_{q} \quad=$ the transportation cost per metre for component $q(q \in Q)$

$m_{q, a, a^{\prime}}=1$ if there is a movement for component $q$ from subarea $a$ to $a^{\prime}\left(a, a^{\prime} \in A\right)$ $=0$ otherwise

$\alpha_{e}, \beta_{e}$, and $\gamma_{e}=$ parameters used by Edge $e(e \in E)$

\section{$\underline{\text { Decision Variables }}$}

$x_{a, s}=$ the $x$-coordinate of left bottom corner of rectangle $s$ representing subarea $a$

$$
\left(a \in A, s \in S_{a}\right) \text {. }
$$

$y_{a, s} \quad=$ the $y$-coordinate of left bottom corner rectangle $s$ representing subarea $a$ $\left(a \in A, s \in S_{a}\right)$.

$u_{a, s} \quad=1$ if rectangle $s$ is used by subarea $a\left(a \in A, s \in S_{a}\right)$, or $=0$ otherwise

$\lambda_{a, a^{\prime}, s, s^{\prime}}=1$ if rectangles $s$ and $s^{\prime}$ are used by subarea $a$ and $a^{\prime}$ respectively

$$
\left(a, a^{\prime} \in A ; s \in S_{a} ; s^{\prime} \in S_{a^{\prime}}\right),
$$

$=0$ otherwise.

$b_{a, a^{\prime}, s, s^{\prime}}^{1}=0$ if rectangle $s$ representing subarea $a$ is on the left hand side of rectangle $s^{\prime}$ representing subarea $a^{\prime}$,

$=1$ otherwise.

$b_{a, a^{\prime}, s, s^{\prime}}^{2}=0$ if rectangle $s$ representing subarea $a$ is on the right hand side of rectangle $s^{\prime}$ representing subarea $a^{\prime}$,

$=1$ otherwise.

$b_{a, a^{\prime}, s, s^{\prime}}^{3}=0$ if rectangle $s$ representing subarea $a$ is above rectangle $s^{\prime}$ representing subarea $a^{\prime}$ $=1$ otherwise. 
$b_{a, a^{\prime}, s, s^{\prime}}^{4}=0$ if rectangle $s$ representing subarea $a$ is below rectangle $s^{\prime}$ representing subarea $a^{\prime}$,

$=1$ otherwise.

$h_{a, s, e}^{1}=0$ if the bottom-left corner point of rectangle $s$ representing subarea $a(a \in \hat{A}$, $\left.s \in S_{a}\right)$ lies on edge $e\left(e \in E_{a}\right)$,

$=1$ otherwise.

$h_{a, s, e}^{2}=0$ if the bottom-right corner point of rectangle $s$ representing subarea $a(a \in \hat{A}$, $\left.s \in S_{a}\right)$ lies on edge $e\left(e \in E_{a}\right)$,

$=1$ otherwise.

$h_{a, s, e}^{3}=0$ if the upper-right corner point of rectangle $s$ representing subarea $a(a \in \hat{A}$, $\left.s \in S_{a}\right)$ lies on edge $e\left(e \in E_{a}\right)$,

$=1$ otherwise.

$h_{a, s, e}^{4}=0$ if the upper-left corner point of rectangle $s$ representing subarea $a(a \in \hat{A}$, $\left.s \in S_{a}\right)$ lies on edge $e\left(e \in E_{a}\right)$,

$=1$ otherwise.

The problem can be formulated as a non-linear MIP model as follows:

Minimise $Z^{c}=\sum_{q \in Q} \sum_{a \in A} \sum_{a^{\prime} \in A} \sum_{s \in S_{a}} \sum_{s^{\prime} \in S_{a^{\prime}}} d_{a, a^{\prime}, s, s^{\prime}} \cdot \lambda_{a, a^{\prime}, s, s^{\prime}} \cdot c_{q} \cdot m_{q, a, a^{\prime}}$

Subject to

$$
\begin{aligned}
& d_{a, a^{\prime}, s, s^{\prime}}=\left|\left(x_{a, s}+\frac{l_{a, s}}{2}\right)-\left(x_{a^{\prime}, s^{\prime}}+\frac{l_{a^{\prime}, s^{\prime}}}{2}\right)\right|+\left|\left(y_{a, s}+\frac{w_{a, s}}{2}\right)-\left(y_{a^{\prime}, s^{\prime}}+\frac{w_{a^{\prime}, s^{\prime}}}{2}\right)\right|, \\
& \forall a, a^{\prime} \in A ; s \in S_{a} ; s^{\prime} \in S_{a^{\prime}} \\
& \sum_{s \in S_{a}} u_{a, s}=1, \forall a \in A \\
& \lambda_{a, a^{\prime}, s, s^{\prime}} \leq u_{a, s}, \forall a=1, \ldots, n-1 ; a^{\prime}=a+1, \ldots, n ; s \in S_{a} ; s^{\prime} \in S_{a^{\prime}} \\
& \lambda_{a, a^{\prime}, s, s^{\prime}} \leq u_{a^{\prime}, s^{\prime}}, \forall a=1, \ldots, n-1 ; a^{\prime}=a+1, \ldots, n ; s \in S_{a} ; s^{\prime} \in S_{a^{\prime}} \\
& \sum_{s \in S_{a}} \sum_{s^{\prime} \in S_{a^{\prime}}} \lambda_{a, a^{\prime}, s, s^{\prime}}=1, \forall a=1, \ldots, n-1 ; a^{\prime}=a+1, \ldots, n ; s \in S_{a} ; s^{\prime} \in S_{a^{\prime}} \\
& \lambda_{a, a^{\prime}, s, s^{\prime}}=\lambda_{a^{\prime}, a, s^{\prime}, s}, \forall a, a^{\prime} \in A ; s \in S_{a} ; s^{\prime} \in S_{a^{\prime}} \\
& \lambda_{a, a^{\prime}, s, s^{\prime}}=0, \forall a \in A, a^{\prime}=a ; s \in S_{a} ; s^{\prime} \in S_{a^{\prime}} \\
& x_{a, s}+l_{a, s} \leq x_{a^{\prime}, s^{\prime}}+M \cdot b_{a, a^{\prime}, s, s^{\prime}}^{1}+M \cdot\left(1-\lambda_{a, a^{\prime}, s, s^{\prime}}\right) \text {, } \\
& \forall a=1, \ldots, n-1 ; a^{\prime}=a+1, \ldots, n ; s \in S_{a} ; s^{\prime} \in S_{a^{\prime}} \\
& x_{a^{\prime}, s^{\prime}}+l_{a^{\prime}, s^{\prime}} \leq x_{a, s}+M \cdot b_{a, a^{\prime}, s, s^{\prime}}^{2}+M \cdot\left(1-\lambda_{a, a^{\prime}, s, s^{\prime}}\right) \text {, } \\
& \forall a=1, \ldots, n-1 ; a^{\prime}=a+1, \ldots, n ; s \in S_{a} ; s^{\prime} \in S_{a^{\prime}}
\end{aligned}
$$




$$
\begin{aligned}
& y_{a, s} \geq y_{a^{\prime}, s^{\prime}}+w_{a^{\prime}, s^{\prime}}-M \cdot b_{a, a^{\prime}, s, s^{\prime}}^{3}-M \cdot\left(1-\lambda_{a, a^{\prime}, s, s^{\prime}}\right), \\
& \forall a=1, \ldots, n-1 ; a^{\prime}=a+1, \ldots, n ; s \in S_{a} ; s^{\prime} \in S_{a^{\prime}} \\
& y_{a^{\prime}, s^{\prime}} \geq y_{a, s}+w_{a, s}-M \cdot b_{a, a^{\prime}, s, s^{\prime}}^{4}-M \cdot\left(1-\lambda_{a, a^{\prime}, s, s^{\prime}}\right) \text {, } \\
& \forall a=1, \ldots, n-1 ; a^{\prime}=a+1, \ldots, n ; s \in S_{a} ; s^{\prime} \in S_{a^{\prime}} \\
& b_{a, a^{\prime}, s, s^{\prime}}^{1}+b_{a, a^{\prime}, s, s^{\prime}}^{2}+b_{a, a^{\prime}, s, s^{\prime}}^{3}+b_{a, a^{\prime}, s, s^{\prime}}^{4}=3 \cdot \lambda_{a, a^{\prime}, s, s^{\prime}}, \\
& \forall a=1, \ldots, n-1 ; a^{\prime}=a+1, \ldots, n ; s \in S_{a} ; s^{\prime} \in S_{a^{\prime}} \\
& \alpha_{e} \cdot x_{a, s}+\beta_{e} \cdot y_{a, s}+\gamma_{e} \leq M \cdot\left(1-u_{a, s}\right), \forall a \in A, s \in S_{a}, e \in E \\
& \alpha_{e} \cdot\left(x_{a, s}+l_{a, s}\right)+\beta_{e} \cdot y_{a, s}+\gamma_{e} \leq M \cdot\left(1-u_{a, s}\right), \forall a \in A, s \in S_{a}, e \in E \\
& \alpha_{e} \cdot\left(x_{a, s}+l_{a, s}\right)+\beta_{e} \cdot\left(y_{a, s}+w_{a, s}\right)+\gamma_{e} \leq M \cdot\left(1-u_{a, s}\right), \forall a \in A, s \in S_{a}, e \in E \\
& \alpha_{e} \cdot x_{a, s}+\beta_{e} \cdot\left(y_{a, s}+w_{a, s}\right)+\gamma_{e} \leq M \cdot\left(1-u_{a, s}\right), \forall a \in A, s \in S_{a}, e \in E \\
& \left(\alpha_{e} \cdot x_{a}+\beta_{e} \cdot y_{a}+\gamma_{e}\right)+h_{a, s, e}^{1} \cdot M+M \cdot\left(1-u_{a, s}\right) \geq 0, \forall a \in \hat{A}, s \in S_{a}, e \in E_{a} \\
& \left(\alpha_{e} \cdot\left(x_{a, s}+l_{a, s}\right)+\beta_{e} \cdot y_{a, s}+\gamma_{e}\right)+h_{a, s, e}^{2} \cdot M+M \cdot\left(1-u_{a, s}\right) \geq 0, \\
& \forall a \in \hat{A}, s \in S_{a}, e \in E_{a} \\
& \left(\alpha_{e} \cdot\left(x_{a, s}+l_{a, s}\right)+\beta_{e} \cdot\left(y_{a, s}+w_{a, s}\right)+\gamma_{e}\right)+h_{a, s, e}^{3} \cdot M+M \cdot\left(1-u_{a, s}\right) \geq 0, \\
& \forall a \in \hat{A}, s \in S_{a}, e \in E_{a} \\
& \left(\alpha_{e} \cdot x_{a, s}+\beta_{e} \cdot\left(y_{a, s}+w_{a, s}\right)+\gamma_{e}\right)+h_{a, s, e}^{4} \cdot M+M \cdot\left(1-u_{a, s}\right) \geq 0, \\
& \forall a \in \hat{A}, s \in S_{a}, e \in E_{a} \\
& h_{a, s, e}^{1}+h_{a, s, e}^{2}+h_{a, s, e}^{3}+h_{a, s, e}^{4} \leq 3 \cdot u_{a, s}, \forall a \in \hat{A}, s \in S_{a}, e \in E_{a} \\
& u_{a, s}=\{0,1\} \quad \forall a \in A, s \in S \\
& \lambda_{a, a^{\prime}, s, s^{\prime}}, b_{a, a^{\prime}, s, s^{\prime}}^{1}, b_{a, a^{\prime}, s, s^{\prime}}^{2}, b_{a, a^{\prime}, s, s^{\prime}}^{3}, b_{a, a^{\prime}, s, s^{\prime}}^{4}=\{0,1\} \forall a, a^{\prime} \in A ; s \in S_{a} ; s^{\prime} \in S_{a^{\prime}} \\
& h_{a, s, e}^{1}, h_{a, s, e}^{2}, h_{a, s, e}^{3}, h_{a, s, e}^{4}=\{0,1\} \forall a \in \hat{A}, s \in S_{a}, e \in E_{a}
\end{aligned}
$$

Where $M$ is an arbitrarily large constant

Objective function (1) aims to minimise the total transportation cost for all components. Constraints (2) calculate the rectilinear distance between two areas for all possible rectangles (dimensions). Constraints (3) guarantee that only one rectangle (dimension) is selected for each subarea. Constraints (4) - (8) determine whether both rectangle $s$ of subarea $a$ and rectangle $s^{\prime}$ of subarea $a^{\prime}$ are selected or not. These constraints define the value of $\lambda_{a, a^{\prime}, s, s^{\prime}}$ for indicating that the transportation cost is calculated based on only the selected rectangles. Constraints (4) - (5) affirm that both rectangles can be selected if one of them is chosen. Constraints (6) make sure that only one rectangle is selected for each subarea. Constraints (7) aim to check the consistency of $\lambda_{a, a^{\prime}, s, s^{\prime}}$ value when the position of indices is different. 
Constraints (8) make sure that variable $\lambda_{a, a^{\prime}, s, s^{\prime}}$ is valid for two different subareas. Constraints (9) - (13) ensure that there is no overlapping among rectangles selected by subareas. These constraints are usually used for rectangle packing problems (Alvarez-Valdes et al., 2013; Chernov et al., 2009). However, we enhance them by considering multiple options for each subarea. Constraints (14) - (17) guarantee that all chosen rectangles are located inside the convex polygon (port area) (Chernov et al., 2009). Constraints (18) - (22) make sure that the at least one corner point of rectangles representing predefined subareas lies on the predefined edges. Constraints (18) - (21) define whether the corner points of selected rectangles (predefined areas) lie on the predefined edges or not whereas Constraints (22) impose that at least one corner point will be located on the predefined edge. Constraints (23) - (25) indicate that the decision variables are binary.

The formulation of the objective function $\left(Z^{c}\right)$ in this model makes the problem nonlinear due to the multiplication of two decision variables $\left(d_{a, a^{\prime}, s, s^{\prime}} \cdot \lambda_{a, a^{\prime}, s, s^{\prime}}\right)$. Here, the problem will be transformed into a linear problem by introducing new decision variables and constraints. Appendix A gives an explanation how the model is transformed into a linear model.

\subsection{The Enhanced MILP (EMILP) model}

The description of the Enhanced MILP (EMILP) model is given in this subsection. This model is built to address the port area with a nonconvex shape, which represents a significant portion of real port shapes. Here we propose an approach to deal with such a problem. The main idea of the approach is to transform the nonconvex polygon into a convex polygon by inserting dummy rectangle areas and forbidden areas of convex polygon shape. Both dummy rectangle areas and forbidden areas are used to prohibit the components from being allocated to the non-existing area of the port. They are realized in the MILP model with different set of constraints. The dummy areas act as virtual subareas where the location of these areas is fixed and other rectangles representing subareas cannot overlap with these areas whereas the forbidden areas aim to avoid locating the corner points of the rectangles (representing subareas) inside these areas.

The use of forbidden areas does not guarantee that the rectangles (representing subareas) will not overlap with the forbidden areas. Therefore, the forbidden areas must be located on a 
corner of port area to ensure that there is no rectangle (representing a subarea) overlapping the forbidden area. In other words, the forbidden area cannot be located in the middle of the port area. Figure 4 presents an example that a forbidden area cannot be used where a dummy rectangle area is added instead of the forbidden area. Figure 4a shows the example of port area that is a nonconvex polygon. In case a forbidden area is inserted, the other area, says Area A, may overlap with the forbidden area as given in Figure 4b. Here, instead of inserting the forbidden area, a rectangle dummy area is added at expense of a slightly smaller port area. In Figure 5a, the area of potential Scottish offshore wind support port, Port Ardersier, as an example is presented. It can be seen clearly that the area of the port is a nonconvex polygon. Figure $5 \mathrm{~b}$ shows the area of the port that has been transformed to a convex polygon shape by inserting three dummy rectangle areas and a forbidden (restricted) area with convex polygon shape.

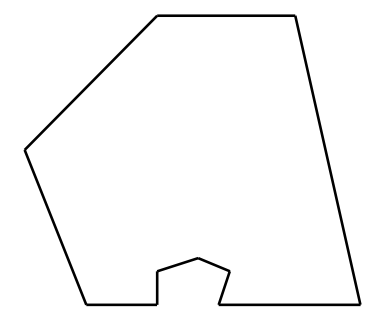

(a)

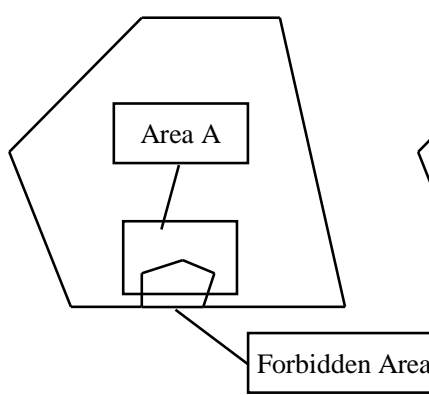

(b)

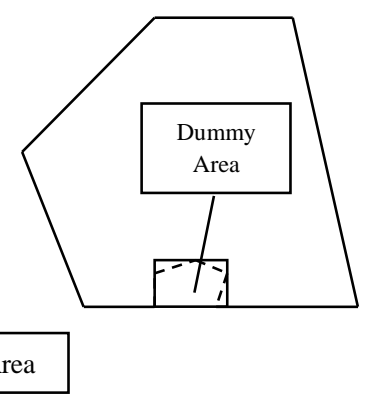

(c)

Figure 4. The example of a forbidden area that cannot be used

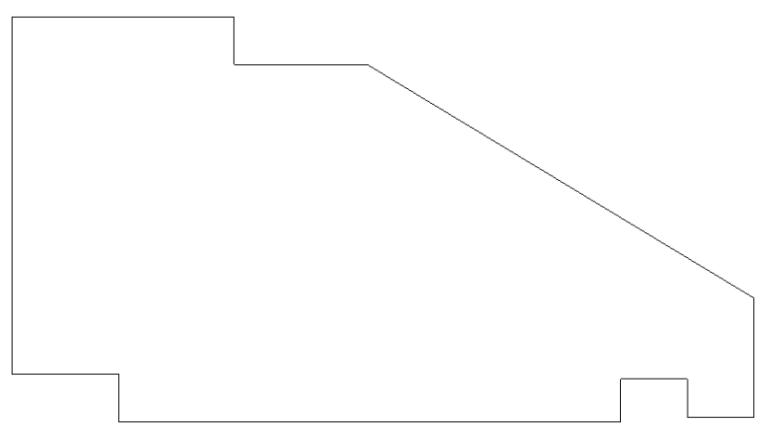

(a)

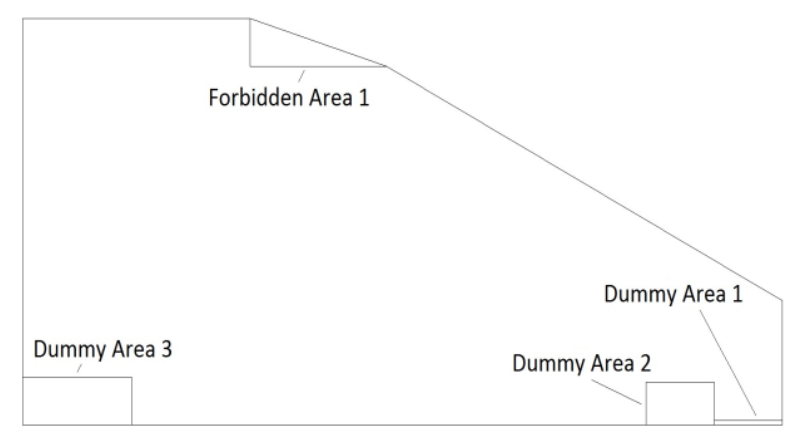

(b)

Figure 5. The area of Port Ardersier

This model also considers the subareas that need to be located in the fixed location. In other words the location of those subareas is fixed which we refer to these areas as fixed subareas. For example the left-bottom corner point of Subarea 1 (Loading Area) must be 
located at $\left(x_{1}^{\prime}, y_{1}^{\prime}\right)$ as this coordinate is a start point of the quay side. In this model, the objective function, decision variables, and constraints are the same as the ones of the BMILP with additional decision variables and constraints to accommodate the presence of the dummy rectangle and forbidden areas.

\section{Dummy Rectangle Areas}

Let $D$ be a set of dummy rectangle areas with $d$ as its index. Each dummy area $d$ has several parameters namely the coordinate of left-bottom corner point of dummy area $d\left(\bar{x}_{d}, \bar{y}_{d}\right)$ and the length $\left(\bar{l}_{d}\right)$ and the width $\left(\bar{w}_{d}\right)$ of the area.

Additional Decision Variables

$\bar{b}_{a, s, d}^{1}=0$ if rectangle $s$ representing subarea $a$ is on the left hand side of dummy area $d$, $=1$ otherwise.

$\bar{b}_{a, s, d}^{2}=0$ if rectangle $s$ representing subarea $a$ is on the right hand side of dummy area $d$, $=1$ otherwise

$\bar{b}_{a, s, d}^{3}=0$ if rectangle $s$ representing subarea $a$ is above dummy area $d$, $=1$ otherwise

$\bar{b}_{a, s, d}^{4}=0$ if rectangle $s$ representing subarea $a$ is below dummy area $d$, $=1$ otherwise

Additional Constraints

$$
\begin{aligned}
& x_{a, s}+l_{a, s} \leq \bar{x}_{d}+M \cdot \bar{b}_{a, s, d}^{1}+M \cdot\left(1-u_{a, s}\right), \forall a \in A, s \in S_{a}, d \in D \\
& \bar{x}_{d}+\bar{l}_{d} \leq x_{a, s}+M \cdot \bar{b}_{a, s, d}^{2}+M \cdot\left(1-u_{a, s}\right), \forall a \in A, s \in S_{a}, d \in D \\
& y_{a, s} \geq \bar{y}_{d}+\bar{w}_{d}-M \cdot \bar{b}_{a, s, d}^{3}-M \cdot\left(1-u_{a, s}\right), \forall a \in A, s \in S_{a}, d \in D \\
& \bar{y}_{d} \geq y_{a, s}+w_{a, s}-M \cdot \bar{b}_{a, s, d}^{4}-M \cdot\left(1-u_{a, s}\right), \forall a \in A, s \in S_{a}, d \in D \\
& \bar{b}_{a, s, d}^{1}+\bar{b}_{a, s, d}^{2}+\bar{b}_{a, s, d}^{3}+\bar{b}_{a, s, d}^{4}=3 \cdot u_{a, s}, \forall a \in A, s \in S_{a}, d \in D \\
& \bar{b}_{a, s, d}^{1}, \bar{b}_{a, s, d}^{2}, \bar{b}_{a, s, d}^{3}, \bar{b}_{a, s, d}^{4}=\{0,1\}, \forall a \in A, s \in S_{a}, d \in D
\end{aligned}
$$

Constraints (26) - (30) guarantee that there is no overlapping between the rectangles (representing subareas) and the dummy areas (Alvarez-Valdes et al., 2013; Chernov et al., 2009). 


\section{Forbidden Areas}

Let $\bar{A}$ be a set of forbidden areas with $\bar{a}$ as its index. Each area $\bar{a}$ has as set of edge vectors $\left(\bar{E}_{\bar{a}}\right)$ indexed by $\bar{e}$. The direction of the edges of the area is the same with the one of port area (clockwise direction). Each edge $\bar{e}\left(e \in \bar{E}_{\bar{a}}\right)$ has parameters $\alpha_{\bar{a}, \bar{e}}, \beta_{\bar{a}, \bar{e}}$, and $\gamma_{\bar{a}, \bar{e}}$ which can be calculated by using the formulation given in Figure 3 .

Additional Decision Variables

$\theta_{a, s, \bar{a}, \bar{e}}^{1}=0$ if the bottom-left corner point of rectangle $s$ representing subarea $a$

$\left(a \in \hat{A}, s \in S_{a}\right)$ lies on the left hand side of edge $\bar{e}\left(e \in \bar{E}_{\bar{a}}\right)$ of forbidden area $\bar{a}$ $(\bar{a} \in \bar{A})$,

$=1$ otherwise.

$\theta_{a, s, \bar{a}, \bar{e}}^{2}=0$ if the bottom-right corner point of rectangle $s$ representing subarea $a$

$\left(a \in \hat{A}, s \in S_{a}\right)$ lies on the left hand side of edge $\bar{e}\left(e \in \bar{E}_{\bar{a}}\right)$ of forbidden area $\bar{a}$ $(\bar{a} \in \bar{A})$,

$=1$ otherwise.

$\theta_{a, s, \bar{a}, \bar{e}}^{3}=0$ if the upper-right corner point of rectangle $s$ representing subarea $a$

$\left(a \in \hat{A}, s \in S_{a}\right)$ lies on the left hand side of edge $\bar{e}\left(e \in \bar{E}_{\bar{a}}\right)$ of forbidden area $\bar{a}$ $(\bar{a} \in \bar{A})$,

$=1$ otherwise.

$\theta_{a, s, \bar{a}, \bar{e}}^{4}=0$ if the upper-left corner point of rectangle $s$ representing subarea $a$

$\left(a \in \hat{A}, s \in S_{a}\right)$ lies on the left hand side of edge $\bar{e}\left(e \in \bar{E}_{\bar{a}}\right)$ of forbidden area $\bar{a}$ $(\bar{a} \in \bar{A})$,

$=1$ otherwise.

Additional Constraints

$$
\begin{aligned}
& \alpha_{\bar{a}, \bar{e}} \cdot x_{a, s}+\beta_{\bar{a}, \bar{e}} \cdot y_{a, s}+\gamma_{\bar{a}, \bar{e}}+M \cdot\left(1-u_{a, s}\right)+M \cdot\left(1-\theta_{a, s, \bar{a}, \bar{e}}^{1}\right) \geq 0, \\
& \quad \forall a \in A, s \in S_{a}, \bar{a} \in \bar{A}, \bar{e} \in \bar{E}_{\bar{a}} \\
& \alpha_{\bar{a}, \bar{e}} \cdot\left(x_{a, s}+l_{a, s}\right)+\beta_{\bar{a}, \bar{e}} \cdot y_{a, s}+\gamma_{\bar{a}, \bar{e}}+M \cdot\left(1-u_{a, s}\right)+M \cdot\left(1-\theta_{a, s, \bar{a}, \bar{e}}^{2}\right) \geq 0, \\
& \quad \forall a \in A, s \in S_{a}, \bar{a} \in \bar{A}, \bar{e} \in \bar{E}_{\bar{a}} \\
& \alpha_{\bar{a}, \bar{e}} \cdot\left(x_{a, s}+l_{a, s}\right)+\beta_{\bar{a}, \bar{e}} \cdot\left(y_{a, s}+w_{a, s}\right)+\gamma_{\bar{a}, \bar{e}}+M \cdot\left(1-u_{a, s}\right)+M \cdot\left(1-\theta_{a, s, \bar{a}, \bar{e}}^{3}\right) \geq 0, \\
& \quad \forall a \in A, s \in S_{a}, \bar{a} \in \bar{A}, \bar{e} \in \bar{E}_{\bar{a}} \\
& \alpha_{\bar{a}, \bar{e}} \cdot x_{a, s}+\beta_{\bar{a}, \bar{e}} \cdot\left(y_{a, s}+w_{a, s}\right)+\gamma_{\bar{a}, \bar{e}}+M \cdot\left(1-u_{a, s}\right)+M \cdot\left(1-\theta_{a, s, \bar{a}, \bar{e}}^{4}\right) \geq 0, \\
& \quad \forall a \in A, s \in S_{a}, \bar{a} \in \bar{A}, \bar{e} \in \bar{E}_{\bar{a}} \\
& \sum_{\bar{e} \in \bar{E}_{\bar{a}} \theta_{a, s, \bar{a}, \bar{e}}^{1} \geq u_{a, s}, \forall a \in A, s \in S_{a}, \bar{a} \in \bar{A}}
\end{aligned}
$$




$$
\begin{aligned}
& \sum_{\bar{e} \in \bar{E}_{\bar{a}}} \theta_{a, s, \bar{a}, \bar{e}}^{2} \geq u_{a, s}, \forall a \in A, s \in S_{a}, \bar{a} \in \bar{A} \\
& \sum_{\bar{e} \in \bar{E}_{\bar{a}}} \theta_{a, s, \bar{a}, \bar{e}}^{3} \geq u_{a, s}, \forall a \in A, s \in S_{a}, \bar{a} \in \bar{A} \\
& \sum_{\bar{e} \in \bar{E}_{\bar{a}}} \theta_{a, s, \bar{a}, \bar{e}}^{4} \geq u_{a, s}, \forall a \in A, s \in S_{a}, \bar{a} \in \bar{A} \\
& \theta_{a, s, \bar{a}, \bar{e}}^{1}, \theta_{a, s, \bar{a}, \bar{e}}^{2}, \theta_{a, s, \bar{a}, \bar{e}}^{3}, \theta_{a, s, \bar{a}, \bar{e}}^{4}=\{0,1\}, \forall a \in A, s \in S_{a}, \bar{a} \in \bar{A}, \bar{e} \in \bar{E}_{\bar{a}}
\end{aligned}
$$

Constraints (32) - (35) make sure that all corner points (4 points) of the selected rectangles representing subareas are outside the forbidden areas. Constraints (36) - (39) indicate that constraints (32) - (35) are applied only for the chosen rectangles. These constraints also ensure that each point of selected rectangles must lie outside the forbidden areas.

\section{Fixed position for fixed subareas}

Let $\tilde{A}$ be a set of fixed subareas where $\tilde{A} \subset A$. The position of left-bottom corner point of the rectangle representing subarea $a(a \in \tilde{A})$ need to be lied at fixed location $\left(x_{a}^{\prime}, y_{a}^{\prime}\right)$. This condition is represented by Constraints (41) and (42).

Additional Constraints

$$
\begin{aligned}
& x_{a, s}=x_{a}^{\prime} \cdot u_{a, s}, \forall a \in \tilde{A}, s \in S_{a} \\
& y_{a, s}=y_{a}^{\prime} \cdot u_{a, s}, \forall a \in \tilde{A}, s \in S_{a}
\end{aligned}
$$

\section{Matheuristic approaches for the port layout problem}

Based on our preliminary study, the MILP models with one possible dimension (rectangle) for each subarea are relatively easy to solve using optimiser software (CPLEX). On the other hand, if the problem size is large where the number of possible rectangles for each subarea is high, the MILP models are very hard to solve using the exact method. To overcome this disadvantage, matheuristic approaches are proposed where a hybridization of a metaheuristic and an exact method is implemented. In this approach, the exact method is embedded into the metaheuristic technique. The metaheuristic approach determines the rectangle (dimension) that will be used for each subarea whereas in the exact method, the MILP model is solved using CPLEX to evaluate the quality of the solution obtained. In other 
words, the exact method only solves the MILP model when the number of possible dimensions for each subarea is such that the resulting problem can be tackled relatively quickly using CPLEX. We refer to this MILP model as the reduced MILP model. The reduced MILP model needs to include dummy rectangle areas, forbidden areas, and fixed subareas when this matheuristic approach is used for solving EMILP problems.

The metaheuristic used in our proposed approaches is based on Variable Neighbourhood Search (VNS). This is due to the fact that VNS is a single solution based heuristic that successfully tackles many combinatorial problems in a shorter computational time compared to its counterpart the population based heuristic (such as genetic algorithms, ant systems, and particle swarm optimization). VNS comprises local search and neighbourhood search. The former looks for local optimality, whereas the latter aims to escape from the local optima. Brimberg and Mladenovic (1996) introduced VNS for solving continuous location-allocation problems. Hansen and Mladenovic (1997) formally formulated VNS for the p-median problem. For more detailed information, Hansen and Mladenovic (2001) and Hansen et al. (2010) describe the implementation of VNS.

In this study, we propose a matheuristic approach based on VNS for solving the installation port layout problem where a new local search for this problem is also introduced. Irawan et al. (2015) also proposed a metaheuristic based on VNS for installation scheduling in offshore wind farms. However, the nature of the problem in the installation scheduling is completely different to the port layout problem that we study here in this paper. Therefore, the procedure of VNS and the local search proposed in this study is not the same as that of Irawan et al. (2015). The main steps of this approach are given by Figure 6 where the first two steps are the initialization step. In Step 1), the best solution and its objective function value are defined whereas in Step 2), three parameters $\left(T, c_{\max }\right.$ and $\left.k_{\max }\right)$ need to be determined. $T$ represents a multi-start approach in our method. In case that $T$ is set to 5 , it means that the method is executed 5 times starting from 5 different initial solutions randomly generated. $c_{\max }$ is the number of runs for the VNS using the best solution obtained $(X)$ as the initial solution. In other words, the procedure of VNS is repeated for $c_{\max }$ iterations. $k_{\max }$ is a parameter to determine the set of neighbourhood structures $\left(\mathcal{N}_{k}\right)$ where $k=1, \ldots, k_{\max }$. 
1) Let $X_{\text {best }}$ be the best set of rectangles representing subareas with $z_{\text {best }}$ denote its objective function value and $\rho_{\text {best }}$ be set of its coordinates of the bottom-left corner for each rectangle.

2) Set $z_{\text {best }}=\infty$ and define $T, c_{\max }$ and $k_{\max }$.

3) Do the following steps $T$ times:

$\underline{\text { Step A }}$

- Choose randomly a rectangle for each subarea as an initial solution $(X)$.

- Solve the reduced MILP model optimally using CPLEX with $X$ as the set of rectangles representing subareas (the number of possible dimensions for each subarea is only one). Let $z$ denote the objective function value and $\rho$ be set of the coordinates of the bottomleft corner for each rectangle. If the solution is infeasible, go back to the previous step.

- Set $X^{\prime} \leftarrow X, z^{\prime} \leftarrow z$, and $\rho^{\prime} \leftarrow \rho$.

$\underline{\text { Step B }}$

Repeat the following steps $c_{\max }$ times.

(I) Set $k=1$

(II) While $k \leq k_{\max }$ do the following steps

(a) Shaking

(* Generate a solution at random from the $k^{\text {th }}$ neighbourhood $*$ )

For $j=1$ to $k$, do the following:

(i). Choose a subarea randomly, say subarea $a$.

(ii). Pick randomly a rectangle in $S_{a}$, say rectangle $s$. If $s=X^{\prime}[a]$ then go back to previous step, otherwise set $s^{\prime}=X^{\prime}[a]$ and $X^{\prime}[a]=s$.

(iii). Solve the reduced MILP model optimally using $X^{\prime}$. Let $z^{\prime}$ and $\rho^{\prime}$ be the objective function value and set of the coordinates respectively.

(iv). If the solution is infeasible, set $X^{\prime}[a]=s^{\prime}$ and go back to Step IIa(i).

End for $j$

(b) Local Search

(i). Set $X^{\prime \prime} \leftarrow X^{\prime}, z^{\prime \prime} \leftarrow z^{\prime}$, and $\rho^{\prime \prime} \leftarrow \rho^{\prime}$

(ii). Apply the local search proposed with $X^{\prime \prime}, z^{\prime \prime}$, and $\rho^{\prime \prime}$ as input/output (see Figure 7).

(c) Move or not

If $z^{\prime \prime}<z$ then

(* Save current solution to be incumbent; return to $\mathcal{N}_{1} *$ )

Set $X \leftarrow X^{\prime \prime}, z \leftarrow z^{\prime \prime}, \rho \leftarrow \rho^{\prime \prime}$, and $k=1$.

Else

(* Current solution is incumbent one; change the neighbourhood $*$ )

Set $X^{\prime} \leftarrow X, z^{\prime} \leftarrow z, \rho^{\prime} \leftarrow \rho$, and $k=k+1$.

$\underline{\text { Step C }}$

If $z<z_{\text {best }}$ then set $X_{\text {best }} \leftarrow X, z_{\text {best }} \leftarrow z$ and $\rho_{\text {best }} \leftarrow \rho$.

4) Take $X_{j}^{\text {best }}$ and $z_{\text {best }}$.

Figure 6. The main steps of the proposed matheuristic based on VNS algorithm 
Step 3) is an iterative (multi-start) process where firstly, the initial solution is generated by choosing at random a rectangle for each subarea. In this approach, the random number is generated using the uniform distribution inversion method. The quality of the solution (objective function value) is attained by solving the reduced MILP model using an exact method (CPLEX). For each initial solution, the VNS is used to improve the solution quality where the procedure of VNS is executed for $c_{\max }$ iteration. Starting from the second iteration, the initial solution of the VNS is obtained from the best solution attained from the previous iteration. In the VNS, the neighbourhood search is performed by 'shaking' the current solution. The shaking process is done by selecting a subarea randomly followed by swapping a rectangle of this subarea (used in current solution) with another randomly chosen rectangle. This process is done $k$ times representing the $k^{\text {th }}$ neighbourhood structures $\left(\mathcal{N}_{k}\right)$ which is adopted from Hansen and Mladenovic (1997).

In the local search, we propose an algorithm based on the interchange heuristic which is presented in Figure 7. In the move or not, a larger neighbourhood is systematically used if an improvement is not found, otherwise the smallest one will be used. In the VNS, the smallest neighbourhood is the one that is closest to the current solution whereas the largest one is farthest from the current solution. This can be done by updating the value of $k$ where $k=1$ represents the smallest neighbourhood whereas $k=k_{\max }$ indicates the largest neighbourhood.

The local search is based on the interchange heuristic using a first improvement strategy. The algorithm aims to find a rectangle $(\breve{s})$ representing a subarea to be swapped with rectangle $s$ used in the current solution. The swap will be done if improvement occurs. In Steps $1 \mathrm{~b}$ and $1 \mathrm{c}(\mathrm{i})$ of Figure 7 , a random approach is used to choose the first subarea and rectangle to be evaluated so the swapping will not always start from the same subarea. This may diversify the search. In Step 1c(ii), the reduced MILP is solved optimally to evaluate the quality of the solution. To speed up the process of finding the optimal solution, an upper bound is fed into the model based on the best objective function (incumbent) value obtained so far. The second step is a termination phase where the local search will stop if there is no improvement after all possible swaps based on incumbent solution have been done. 


\section{Procedure Local Search $\left(X^{\prime \prime}, z^{\prime \prime}, \rho^{\prime \prime}\right)$}

\section{Step 1}

a. Set $\theta=0$ ( $\theta$ is the saving occurred from swapping) and $\sigma=$ false.

b. Choose randomly a subarea from set $A$, say subarea $\vec{a}$.

c. For $i=1$ to $|A|$, do the following:

(i) Pick randomly a rectangle from set $S_{\breve{a}}$, say shape $\breve{s}$

(ii) For $j=1$ to $\left|S_{\breve{a}}\right|$, do the following steps:

○ Set $\breve{X} \leftarrow X^{\prime \prime}$.

○ Set $\breve{X}[\breve{a}] \leftarrow \breve{s}$.

- Solve the reduced MILP model optimally using $\breve{X}$ as the set of rectangles representing subarea. In the reduced MILP, $z^{\prime \prime}$ is used as an upper bound to speed up the computing time. Let $\breve{z}$ denote its objective function value and $\breve{\rho}$ be set of the coordinates of the bottom-left corner for each rectangle.

○ Set $\theta \leftarrow z^{\prime \prime}-\bar{z}$

○ If $\theta>0$ and the solution is feasible, do the followings:

- Set $X^{\prime \prime} \leftarrow \breve{X}, z^{\prime \prime} \leftarrow \breve{z}$, and $\rho^{\prime \prime} \leftarrow \breve{\rho}$.

- Go to Step 2.

○ Set $\breve{s} \leftarrow \breve{s}+1$

○ If $\breve{s}>\left|S_{\breve{a}}\right|$, set $\breve{s} \leftarrow 1$.

End for $j$

(iii) Set $\breve{a} \leftarrow \breve{a}+1$.

(iv) If $\breve{a}>|A|$, set $\hat{a} \leftarrow 1$

End for $i$

\section{Step 2}

- If $\theta \leq 0$ then stop, otherwise go to Step 1 .

Figure 7. The local search for solving the port layout problems

\section{Computational Study}

Computational experiments were carried out to assess the performance of the proposed solution approaches. The code was written in C++ .Net 2012 and used the IBM ILOG CPLEX version 12.6 Concert Library (for exact method). The experiments were run on a PC with an Intel Core i5 CPU @ 3.20GHz processor, 8.00 GB of RAM and under Windows 7. In the computational experiments, two types of dataset are used. In the first dataset (Dataset 1), the port area with convex irregular polygon shape is generated randomly to assess our approaches in solving the BMILP problem. In the second one (Dataset 2), the area of Port Ardersier is used to demonstrate the EMILP problem as the shape of the port area is a nonconvex irregular polygon. 


\subsection{Experiments on the randomly generated port area (Dataset 1)}

\section{A. Data}

The data are generated randomly based on approximations for the purpose of conducting computational experiments. In this study, three main components of an offshore wind turbine are considered namely tower, nacelle, and blade. Table 3 shows the transportation cost for each component.

Table 3. List of components

\begin{tabular}{ccc}
\hline Component ID & Component Name & Transportation cost $(£ / \mathrm{m})$ \\
\hline C1 & Tower & 10.5 \\
C2 & Nacelle & 5.2 \\
C3 & Blade & 8.3 \\
\hline
\end{tabular}

The installation port area dedicated for the OWF is divided into eight types of subarea in this dataset, which is presented in Table 4. The loading area is the area located on the quay side where the components need to be loaded / unloaded into/from a vessel. In this subarea, heavy lift cranes are installed. The stage areas (tower, nacelle, and blade stages) are the place where the components are laid down waiting to be transferred into the vessel. Components that have been manufactured will be kept in the storage areas. The unloading area is usually near to the main road/rail entrance where wind turbine components produced by inland manufacturers are transported by road/rail. Once the components have been unloaded, they are transferred to the storage areas. In Table 4, five possible rectangles are presented with their dimension, length $(l)$ and width $(w)$.

Table 4. List of subareas with their possible rectangles for Dataset 1

\begin{tabular}{|c|c|c|c|c|c|c|c|c|c|c|c|}
\hline \multirow{2}{*}{$\begin{array}{c}\text { Area } \\
\text { ID }\end{array}$} & \multirow{2}{*}{ rea Name } & \multicolumn{2}{|c|}{ Rectangle 1} & \multicolumn{2}{|c|}{ Rectangle 2} & \multicolumn{2}{|c|}{ Rectangle 3} & \multicolumn{2}{|c|}{ Rectangle 4} & \multicolumn{2}{|c|}{ Rectangle 5} \\
\hline & & $l$ & $w$ & $l$ & $w$ & $l$ & $W$ & $l$ & $w$ & $l$ & $w$ \\
\hline A1 & & 0.00 & 200.00 & 00.00 & 250.00 & 38.00 & 210.08 & 210.08 & 238.00 & 246.00 & 203.25 \\
\hline A2 & Stage & 0.00 & 72.00 & 2.00 & 200.00 & 240.00 & 60.00 & 0.00 & 240.00 & 192.00 & 75.00 \\
\hline A3 & & & & 12.00 & & 240.00 & 60.00 & 00.00 & & 192.00 & 75.00 \\
\hline A4 & Blade Stage & 200.00 & 338.00 & 338.00 & 200.00 & 212.00 & 318.87 & 318.87 & 212.00 & 208.00 & 325.00 \\
\hline A5 & & 200.00 & & 160.00 & & 250.00 & 160.00 & 212.00 & & 188.68 & 212.00 \\
\hline A6 & & 200.00 & $2+.00$ & 24.00 & 200.00 & 192.00 & 25.00 & 25.00 & 192.00 & 160.00 & 30.00 \\
\hline A7 & & 200.00 & 70.00 & 70.00 & 200.00 & 175.00 & 80.00 & 80.00 & 175.00 & 160.00 & 87.50 \\
\hline A8 & Unloading Area & 250.00 & 150.00 & 150.00 & 250.00 & 240.00 & 156.25 & 156.25 & 240.00 & 200.00 & 187.50 \\
\hline
\end{tabular}


Two port areas of irregular convex polygon shape are generated which are referred to as Polygon PS and PL. The size of Polygon PL area is slightly bigger than the one of PS. Table 5 shows the coordinates for each point that make up the polygons where the edges are constructed by drawing a straight line using a pair of points $(1-2,2-3, \ldots, 13-1)$. In total both polygons have 13 vertices. The quay side is located at the $\mathrm{x}$-axis line where $y=0$. Table 6 presents the parameter values $(\alpha, \beta, \gamma)$ for each edge for both Polygon PS and PL where these value are calculated using the formulation given in Figure 3. In total, there are 13 edges for each polygon. The list of subareas in which at least one their corner points lies on the predefined edges is given in Table 7. For Polygons PS and PL, Edge E13 is the border of the quay side whereas Edge E6 is the nearest side to main road/rail. In other words, loading area (A1) must lie on Edge E13 whilst the unloading area (A8) is recommended to be located near to Edge E6.

Table 5. List of the coordinates of the vertices of the polygons for Dataset 1

\begin{tabular}{|c|c|c|c|c|}
\hline \multirow{2}{*}{ Vertices } & \multicolumn{2}{|c|}{ Polygon PS } & \multicolumn{2}{|c|}{ Polygon PL } \\
\hline & $x$ & $y$ & $X$ & $y$ \\
\hline Vertices 1 & 30 & 0 & 30 & 0 \\
\hline Vertices 2 & 0 & 150 & 0 & 150 \\
\hline Vertices 3 & 0 & 300 & 0 & 300 \\
\hline Vertices 4 & 10 & 400 & 10 & 400 \\
\hline Vertices 5 & 20 & 550 & 20 & 550 \\
\hline Vertices 6 & 60 & 600 & 60 & 700 \\
\hline Vertices 7 & 400 & 600 & 400 & 700 \\
\hline Vertices 8 & 500 & 575 & 600 & 650 \\
\hline Vertices 9 & 600 & 500 & 700 & 500 \\
\hline Vertices 10 & 700 & 350 & 800 & 350 \\
\hline Vertices 11 & 700 & 40 & 800 & 40 \\
\hline Vertices 12 & 600 & 30 & 700 & 30 \\
\hline Vertices 13 & 300 & 0 & 300 & 0 \\
\hline
\end{tabular}

Table 8 indicates the movement of the components of the wind turbine in the port area. Tower $(\mathrm{C} 1)$ travels from the unloading area to the tower storage area. If this component is needed for the installation in the offshore wind farm, it will be transferred to tower stage area waiting to be loaded to the vessel, which will be performed in the loading area. Similar movements occur for nacelle (C2) and blade (C1). In general, the movement of components starts from the unloading area to the storage area. From the latter area, the components will be transferred to the stage area before they are transported to loading area. 
Table 6. List of the edges of the polygons for Dataset 1

\begin{tabular}{lccccccc}
\hline \multirow{2}{*}{ Edge } & \multicolumn{3}{c}{ Polygon PS } & & \multicolumn{3}{c}{ Polygon PL } \\
\cline { 2 - 4 } \cline { 6 - 8 } & $\alpha$ & $\beta$ & $\gamma$ & & $\alpha$ & $\beta$ & $\gamma$ \\
\hline E1 & -0.981 & -0.196 & 29.417 & & -0.981 & -0.196 & 29.417 \\
E2 & -1.000 & 0.000 & 0.000 & & -1.000 & 0.000 & 0.000 \\
E3 & -0.995 & 0.100 & -29.851 & & -0.995 & 0.100 & -29.851 \\
E4 & -0.998 & 0.067 & -16.630 & & -0.998 & 0.067 & -16.630 \\
E5 & -0.781 & 0.625 & -327.965 & & -0.966 & 0.258 & -122.390 \\
E6 & 0.000 & 1.000 & -600.000 & & 0.000 & 1.000 & -700.000 \\
E7 & 0.243 & 0.970 & -679.100 & & 0.243 & 0.970 & -776.114 \\
E8 & 0.600 & 0.800 & -760.000 & & 0.832 & 0.555 & -859.785 \\
E9 & 0.832 & 0.555 & -776.580 & & 0.832 & 0.555 & -859.785 \\
E10 & 1.000 & 0.000 & -700.000 & & 1.000 & 0.000 & -800.000 \\
E11 & 0.100 & -0.995 & -29.851 & & 0.100 & -0.995 & -39.801 \\
E12 & 0.100 & -0.995 & -29.851 & & 0.075 & -0.997 & -22.437 \\
E13 & 0.000 & -1.000 & 0.000 & & 0.000 & -1.000 & 0.000 \\
\hline
\end{tabular}

Table 7. List of subareas that touch edges for Dataset 1

\begin{tabular}{ccc}
\hline Area ID & Area Name & Edges \\
\hline A1 & Loading Area & E13 \\
A8 & Unloading Area & E6 \\
\hline
\end{tabular}

Table 8. The movement of the components for Dataset 1

\begin{tabular}{ccc}
\hline \multirow{2}{*}{ Component ID } & \multicolumn{2}{c}{ Area ID } \\
\cline { 2 - 3 } & From & To \\
\hline C1 & A8 & A5 \\
C1 & A5 & A2 \\
C1 & A2 & A1 \\
C2 & A8 & A6 \\
C2 & A6 & A3 \\
C2 & A3 & A1 \\
C3 & A8 & A7 \\
C3 & A7 & A4 \\
C3 & A4 & A1 \\
\hline
\end{tabular}

\section{B. Results}

In the computational experiments, the two methods (exact method and Matheuristic approach) are used to solve the BMILP problem using Polygons PS and PL. We also vary the number of areas that touch the polygon to 1 (AF1) and 2 (AF2). In AF1, at least one corner point of Area A1 lies on Edge 13 whereas in AF2, Area A1 and A8 touch Edge E13 and E6 respectively. In other words, there are 4 main problems, namely PS-AF1, PS-AF2, PL-AF1, 
and PL-AF2. In the experiments, the number of possible rectangles for each subarea is set to $\eta$ where $\eta=\left|S_{a}\right|, a=1, \ldots,|A|$ meaning that the number of possible rectangles for a subarea is the same with other subareas. For each main problem, we also vary the value of $\eta$ from 2 to 5. In case $\eta=2$, the first two rectangles in Table 4 is considered while $\eta=4$, the first four are taken into account.

To assess our proposed matheuristic approach, we compare the solutions of the proposed method with those of the exact method using IBM ILOG CPLEX version 12.6 Concert Library. As the problems are very hard to solve by exact method, we limit the computational time (CPU) to three hours so the lower bound (LB) and upper bound (UB) can be attained. The performance of the proposed matheuristic approach will also be measured by \%Gap between the $Z$ value obtained by the matheuristic approach and the lower bound (LB) obtained from the exact method. \%Gap is calculated as follows:

$$
\% \text { Gap }=\frac{Z_{m}-L B}{Z_{m}} \times 100
$$

where $Z_{m}$ refers to the feasible solution cost obtained by either the exact method (UB) or the matheuristic method.

In the matheuristic approach, two scenarios are implemented where in the first scenario, the value of parameters $T, c_{\max }$ and $k_{\max }$ are set to $1, \eta$ and $|A|$ respectively whereas in the second one, we set those parameters to 5, 1 and $|A|$ respectively. The first scenario tends to improve the best solution obtained so far by executing the VNS $\eta$ times. The second one applies a multi-start approach where a set of initial solutions is randomly generated. Each initial solution is improved by implementing the VNS once. The best solution is the one with the smallest objective function value. Table 9 presents the computational results on the randomly generated port area.

In Table 9, the results of the exact method (UB, LB, \% Gap and CPU time in seconds) are given. In our experiments, to assess the consistency of the proposed metaheuristic methods, in each instance the methods were executed 5 times therefore the average result (Avg Z) as well as the best one (Best Z) are provided. According to Table 9, the objective function value improves when the number of possible rectangles included in the model increases as there is a greater number of possible options when choosing the area dimension. The CPU time rises 
exponentially along with the number of possible rectangles. It is also noted that for some instances, CPLEX cannot guarantee optimality (\%Gap > 0) after the set time of 3 hours.

The objective function values on a bigger polygon (PL) are better than the ones on the smaller one (PS) as more available spaces gives less restrictions. Similarly, when we restrict two areas (AF2) that need to touch the border of polygon, the objective function value is worse than one area (AF1). However, instance PL-AF1 is the most difficult to solve by the exact method (CPLEX) with a longer computational time needed to solve this instance. Based on the average of CPU time, the matheuristic approaches run much faster than the exact method to solve the problems. It is noted that regarding the average gap, the proposed matheuristic approach (both scenarios) yields a smaller gap compared to the exact method using CPLEX (run within 3 hours). The matheuristic approach using Scenario 2 produces a smaller gap than the one using Scenario 1. However, the average computational time required by scenario 2 to solve the problems is 1.6 times the one needed by scenario 1 . In general, for this case the approach using scenario 2 performs better than the one using scenario 1 at the expense of a longer computational time.

Figure 8 shows the examples of the optimal port layout generated by the exact method. Figures $8 \mathrm{a}$ and $8 \mathrm{~b}$ show the port layout on instances PS-AF1 and PS-AF2 respectively where both instances use 3 possible rectangles for each area. From Figure 8, it is shown that Area A1 (loading area) lies on the bottom border of polygon which represents the quay side. In Figure 8b, PS-AF2 instance, Area A8 (unloading area) needs to touch Edge E6, which is near to the main road/rail entrance. On the other hand, for PS-AF1 instance (Figure 8a), Area A8 is located in the middle of the port area as there is no restriction for this area.

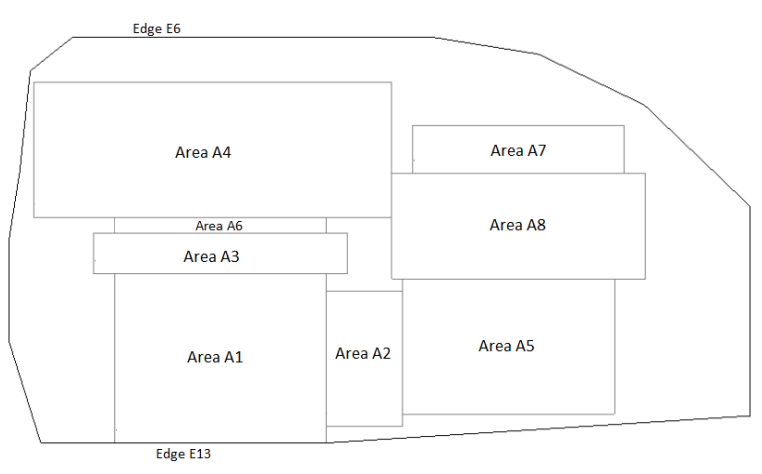

(a)

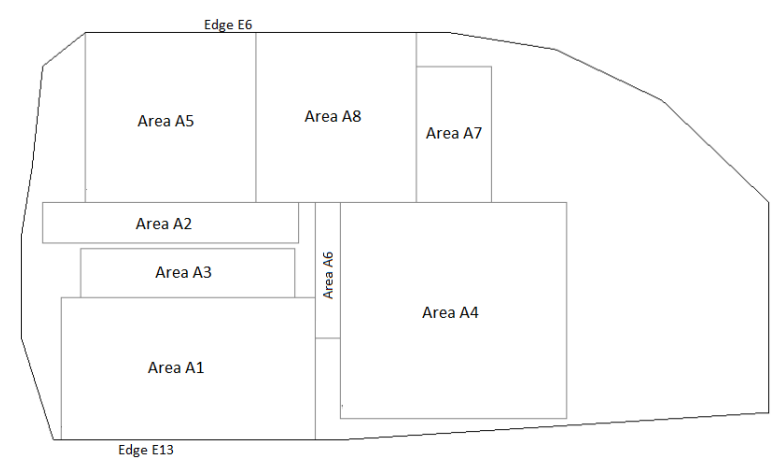

(b)

Figure 8 . The examples of the port layout generated by exact method 
Table 9. The results on the BMILP problem using the randomly generated port area (Dataset 1)

\begin{tabular}{|c|c|c|c|c|c|c|c|c|c|c|c|c|c|c|c|}
\hline \multirow{3}{*}{$\begin{array}{l}\text { Ins- } \\
\text { tance }\end{array}$} & \multirow{3}{*}{$\begin{array}{l}\text { Num. } \\
\text { of } \\
\text { shapes }\end{array}$} & \multicolumn{4}{|c|}{ Exact Method } & \multicolumn{5}{|c|}{ Matheuristic Based on VNS Scenario 1} & \multicolumn{5}{|c|}{ Matheuristic Based on VNS Scenario 2} \\
\hline & & \multirow{2}{*}{ UB } & \multirow{2}{*}{ LB } & \multirow{2}{*}{ Gap (\%) } & \multirow{2}{*}{$\mathrm{CPU}$} & \multicolumn{2}{|c|}{ Best Result } & \multicolumn{3}{|c|}{ Average Result } & \multicolumn{2}{|c|}{ Best Result } & \multicolumn{3}{|c|}{ Average Result } \\
\hline & & & & & & $\mathrm{Z}$ & Gap (\%) & $\mathrm{Z}$ & Gap (\%) & $\mathrm{CPU}$ & $\mathrm{Z}$ & Gap (\%) & $\mathrm{Z}$ & Gap (\%) & $\mathrm{CPU}$ \\
\hline \multirow{4}{*}{ PSAF1 } & 2 & $13,625.40$ & $13,625.40$ & 0.0000 & 113.94 & $13,625.40$ & 0.0000 & $13,625.40$ & 0.0000 & 33.59 & $13,625.40$ & 0.0000 & $13,625.40$ & 0.0000 & 131.03 \\
\hline & 3 & $13,470.34$ & $13,470.34$ & 0.0000 & $4,664.78$ & $13,470.34$ & 0.0000 & $13,496.58$ & 0.1944 & 104.38 & $13,470.34$ & 0.0000 & $13,470.34$ & 0.0000 & 274.06 \\
\hline & 4 & $13,231.51$ & $4,103.70$ & 68.9854 & $11,108.81$ & $13,231.51$ & 68.9854 & $13,266.79$ & 69.0679 & 232.05 & $13,231.51$ & 68.9854 & $13,254.94$ & 69.0402 & 367.67 \\
\hline & 5 & $13,729.45$ & $2,040.57$ & 85.1373 & $10,801.92$ & $13,061.05$ & 84.3767 & $13,061.05$ & 84.3767 & 407.27 & $13,061.05$ & 84.3767 & $13,061.05$ & 84.3767 & 597.97 \\
\hline \multirow{4}{*}{ PSAF2 } & 2 & $14,468.40$ & $14,468.40$ & 0.0000 & 26.41 & $14,468.40$ & 0.0000 & $14,468.40$ & 0.0000 & 18.95 & $14,468.40$ & 0.0000 & $14,468.40$ & 0.0000 & 57.39 \\
\hline & 3 & $14,376.99$ & $14,376.99$ & 0.0000 & 464.32 & $14,376.99$ & 0.0000 & $14,421.56$ & 0.3090 & 55.04 & $14,376.99$ & 0.0000 & $14,377.59$ & 0.0042 & 117.17 \\
\hline & 4 & $14,188.14$ & $14,188.14$ & 0.0000 & $5,097.00$ & $14,188.14$ & 0.0000 & $14,188.14$ & 0.0000 & 105.08 & $14,188.14$ & 0.0000 & $14,188.14$ & 0.0000 & 186.61 \\
\hline & 5 & $14,453.60$ & $4,495.60$ & 68.8963 & $10,802.20$ & $14,074.14$ & 68.0577 & $14,074.14$ & 68.0577 & 169.32 & $14,074.14$ & 68.0577 & $14,074.14$ & 68.0577 & 251.11 \\
\hline \multirow{4}{*}{ PLAF1 } & 2 & $13,620.00$ & $13,620.00$ & 0.0000 & 268.56 & $13,620.00$ & 0.0000 & $13,627.20$ & 0.0528 & 67.49 & $13,620.00$ & 0.0000 & $13,620.00$ & 0.0000 & 196.57 \\
\hline & 3 & $13,465.27$ & $13,465.27$ & 0.0000 & $9,751.57$ & $13,465.27$ & 0.0000 & $13,469.32$ & 0.0301 & 160.07 & $13,465.27$ & 0.0000 & $13,467.30$ & 0.0150 & 330.97 \\
\hline & 4 & $13,319.60$ & $6,892.30$ & 48.2545 & $10,802.20$ & $13,231.51$ & 47.9100 & $13,245.64$ & 47.9655 & 299.06 & $13,231.51$ & 47.9100 & $13,262.64$ & 48.0322 & 581.30 \\
\hline & 5 & $13,362.39$ & $2,814.00$ & 78.9409 & $10,800.44$ & $13,061.05$ & 78.4550 & $13,091.09$ & 78.5045 & 560.94 & $13,061.05$ & 78.4550 & $13,091.09$ & 78.5045 & 790.12 \\
\hline \multirow{4}{*}{ PLAF2 } & 2 & $15,267.00$ & $15,267.00$ & 0.0000 & 36.55 & $15,267.00$ & 0.0000 & $15,267.00$ & 0.0000 & 32.02 & $15,267.00$ & 0.0000 & $15,267.00$ & 0.0000 & 59.56 \\
\hline & 3 & $15,267.00$ & $15,267.00$ & 0.0000 & 387.65 & $15,267.00$ & 0.0000 & $15,267.00$ & 0.0000 & 76.23 & $15,267.00$ & 0.0000 & $15,267.00$ & 0.0000 & 100.45 \\
\hline & 4 & $15,267.00$ & $15,158.10$ & 0.7133 & $10,800.13$ & $15,267.00$ & 0.7133 & $15,267.00$ & 0.7133 & 146.20 & $15,267.00$ & 0.7133 & $15,267.00$ & 0.7133 & 162.36 \\
\hline & 5 & $15,267.00$ & $7,859.59$ & 48.5191 & $10,801.02$ & $15,267.00$ & 48.5191 & $15,267.00$ & 48.5191 & 187.75 & $15,267.00$ & 48.5191 & $15,267.00$ & 48.5191 & 201.42 \\
\hline Average & & & & 24.9654 & $6,045.47$ & & 24.8136 & & 24.8619 & 165.96 & & 24.8136 & & 24.8289 & 275.36 \\
\hline
\end{tabular}




\subsection{Port Ardersier Example (Dataset 2)}

\section{A. Port area data}

The approximated area of Port Ardersier can be seen in Figure 5, where the shape of the port area is an irregular nonconvex polygon. In this case, we deal with the EMILP problem. Table 10 shows the coordinates of Port Ardersier vertices which the port area has been transformed into a convex polygon. Similar to the previous experiments, the quay side is at the $\mathrm{x}$-axis line. In Table 11, a list of dummy areas is given where Columns 2 and 3 indicate the $\mathrm{x}$ - and $\mathrm{y}$ - coordinate of bottom-left corner point of the dummy area that need to be located. The table also provides the length and the width of each dummy rectangle area.

Table 10. List of the coordinates of the vertices of the port area (Dataset 2)

\begin{tabular}{lcc}
\hline Vertices & $X$ & $y$ \\
\hline Vertices 1 & 0 & 0 \\
Vertices 2 & 0 & 850 \\
Vertices 3 & 500 & 850 \\
Vertices 4 & 800 & 750 \\
Vertices 5 & 1670 & 260 \\
Vertices 6 & 1670 & 0 \\
\hline
\end{tabular}

Table 11. List of dummy areas (Dataset 2)

\begin{tabular}{lcccc}
\hline Dummy Area & $x$ & $Y$ & Length & width \\
\hline Dummy 1 & 1520 & 0 & 150 & 10 \\
Dummy 2 & 1370 & 0 & 150 & 90 \\
Dummy 3 & 0 & 0 & 240 & 100 \\
\hline
\end{tabular}

Table 12 shows a list of the forbidden area coordinates where the number of points that make up the polygon is given. The construction of the edge vector is similar to the one of the convex polygon (port area). The formulation in Figure 3 is also used to calculate the parameters $(\alpha, \beta$, and $\gamma)$ for each edge.

Table 12. List of forbidden area (Dataset 2)

\begin{tabular}{cccc}
\hline Forbidden area & \#Points & $X$ & $y$ \\
\hline \multirow{3}{*}{ Forb. area 1 } & & 500 & 750 \\
& \multirow{2}{*}{3} & 500 & 850 \\
& & 800 & 750 \\
\hline
\end{tabular}


In this case study, the Loading Area, which needs to be located at the quay side, is treated as a fixed subarea where the position of bottom-left corner point of this subarea is located at coordinate $(240,0)$. In addition, the Unloading Area needs also to touch Edge E5 (the border on right side of the polygon) as this border is the nearest one to main road/rail entrance. The computational experiments on two instances were carried out. The first instance (Instance 2a) consists of 8 subareas and 3 components where each subarea has up to 5 possible dimensions. The second instance (Instance $2 \mathrm{~b}$ ) is a relatively large problem where it comprises 12 subareas and 5 components with each subarea has up to 10 possible dimensions.

The exact method and the proposed matheuristic approach are also used to address this EMILP problem. In the exact method, CPLEX was also used to solve the problem. We limit the computational time to 3 hours to obtain upper and lower bounds which is similar to the previous experiments. In the matheuristic approach, the scenario along with parameter setting used is also the same as in the previous experiments. For each instance the methods were also run 5 times to assess the consistency of our proposed method.

\section{B. Computational Results on Instance 2a}

In this experiment, the subareas and components considered along with its transportation cost and its movement are the same with the previous experiment. We also vary the number of possible rectangles for each subarea $(\eta)$ from 2 to 5 where the detailed data is given in Table B1 of Appendix B. The computational results on Instance 2a are presented in Table 13 where the exact method was able to guarantee optimality for the first three problems. For the fourth problem ( 5 possible dimensions), the \%Gap obtained by CPLEX is relatively high which is $40.8509 \%$. Running CPLEX for 35 hours for the fourth problem produced a guaranteed optimal solution equivalent to the upper bound value $(30,743.75)$ shown in Table 13.

Based on Table 13, similarly to the previous experiments, the matheuristic approach runs much faster than the exact method. When 5 possible rectangles in used, the matheuristic approach with Scenarios 1 and 2 need only around 3 and 4 minutes respectively to solve the problem. The value of $Z$ for the problem with $\eta=4$ and $\eta=5$ is the same meaning that an additional rectangle for each area does not improve the quality of the solution. By executing the matheuristic methods five times, the optimal solutions can be obtained as the Gap (\%) of 
both metaheuristic scenarios is the same as the one of the exact method. According to the average gap, the matheuristic with Scenario 2 produces a smaller gap compared to the one with Scenario 1. These results confirm the results of the previous experiments where in general, the matheuristic with Scenario 2 produces better solutions compared to the one with Scenario 1. However, the average computational time needed by the matheuristic with Scenario 2 to solve the problems is almost double the one needed by the matheuristic with Scenario 1.

Figure 9 presents the examples of the optimal layout for Port Ardersier generated by the exact method on Instance 2a. Figures $9 \mathrm{a}$ and $9 \mathrm{~b}$ show the port layout with 1 and 4 possible rectangles respectively. From the figure, it is shown that Area A1 (loading area) lies on the quay side whereas Area A8 is located on the right side of area near to the main road/rail. The figure also reveals that Areas A1-A8 do not overlap with dummy areas and forbidden areas.

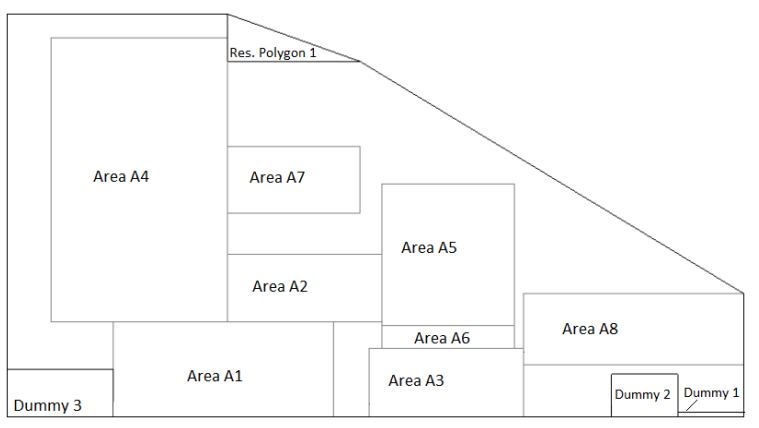

(a)

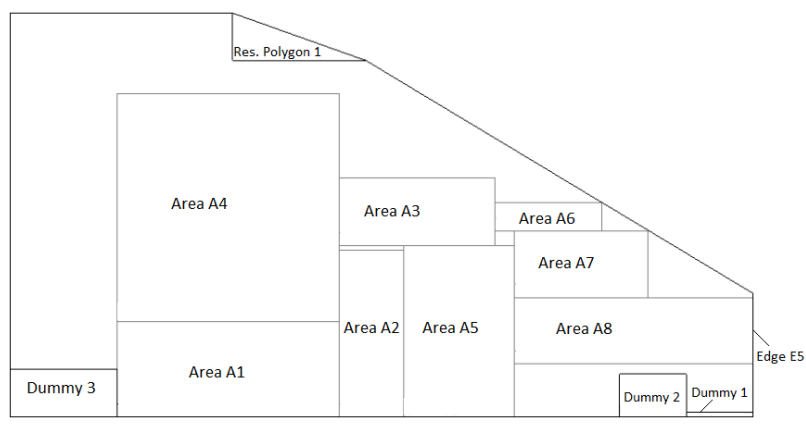

(b)

Figure 9. The examples of Port Ardersier layout produced by the exact method

\section{Computational Results on Instance 2b}

An offshore wind turbine consists of 5 main components if the foundation and transition piece are included. In Instance $2 \mathrm{~b}$, all the 5 components are considered. As each component needs storage and stage areas, there are 12 subareas taken into account in this case study including loading and unloading areas. In other words, the installation port layout for an offshore wind farm is unlikely to have more than 12 subareas. The movement of each component is the same as the previous experiment where a component will be transferred from unloading area to storage area, then transported to stage area, and finally moved to loading area. The transportation cost of tower, nacelle and blades is the same to the previous experiments where the transportation cost of foundation and transition piece is set to $£ 13 / \mathrm{m}$ 
and $£ 6 / \mathrm{m}$ respectively. We vary the number of possible rectangles for each subarea $(\eta)$ from 6 to 10 where the detailed data is given in Table B2 of Appendix B.

According our experiments on this instance, the exact method using CPLEX was not able to obtain the lower bound within 3 hours. In other words, the value of LB is still zero. However, CPLEX managed to obtain the upper bound (UB) value. The performance of the proposed matheuristic approach will be assessed by \%Dev which is calculated as follows:

$$
\% \text { Dev }=\frac{Z_{m}-Z^{\prime}}{Z_{m}} \times 100
$$

where

$Z_{m}$ : the feasible solution cost obtained by either the exact method (UB) or the matheuristic method.

$Z^{\prime}$ : the best known solution cost.

In the proposed matheuristic approach, we only use Scenario 1 in these experiments with the computational time limited to 1 hour. We use this scenario as it runs faster than its counterpart, Scenario 2. We also make a further enhancement inside the local search procedure proposed in Figure 7. As the number of subareas in this case study is relatively large, to speed up the search process, in Step 1c(ii) of Figure 7 the solution of the reduced MILP problem produced by CPLEX is not required to be guaranteed optimal. In the reduced problem, CPLEX may find the optimal solution although the \%Gap between UB and LB is not zero. In this problem, it seems that a good LB is more difficult to obtain using CPLEX than a good UB. Therefore, in in Step 1c (ii) of Figure 7 for this instance, we add \%Gap as a termination criterion for CPLEX to solve the reduced MILP which we set \%Gap to $5 \%$.

Table 14 shows the computational results on Instance $2 \mathrm{~b}$ where the matheuristic approach produces better solutions than the exact method for all problems while requiring a smaller computing time. The exact method yields a deviation of $5.4748 \%$ whereas the average deviation of the proposed matheuristic using Scenario 1 is $0.7 \%$. Moreover, the best solution found for all problems in this instance is also produced by the matheuristic approach (by executing the matheuristic methods five times). 
Table 13. The results on the EMILP problem using the area of Port Ardersier (Instance 2a)

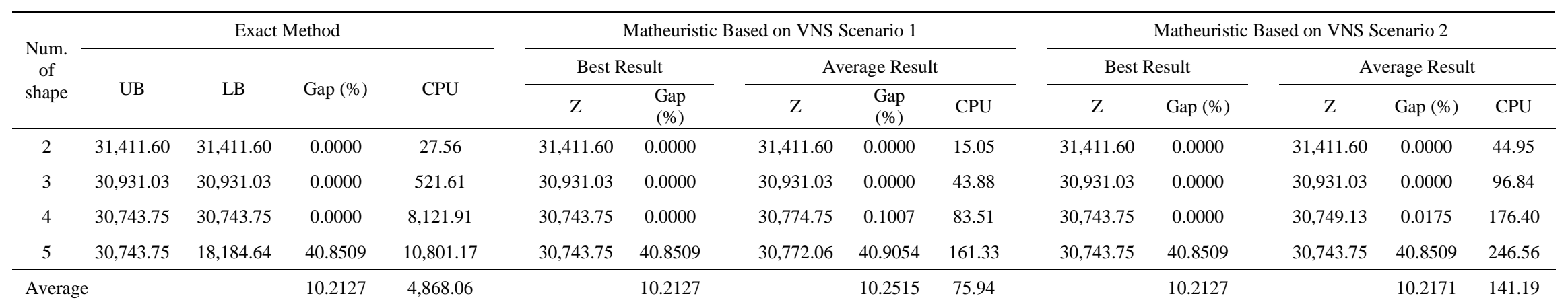

Table 14. The results on the EMILP problem using the area of Port Ardersier (Instance 2b)

\begin{tabular}{|c|c|c|c|c|c|c|c|c|c|}
\hline \multirow{3}{*}{$\begin{array}{l}\text { Number } \\
\text { of shapes }\end{array}$} & \multirow{3}{*}{$\begin{array}{c}\text { Best } \\
\text { Known } Z \\
\left(Z^{\prime}\right)\end{array}$} & \multicolumn{3}{|c|}{ Exact Method } & \multicolumn{5}{|c|}{ Matheuristic Based on VNS Scenario 1} \\
\hline & & \multirow{2}{*}{ UB } & \multirow{2}{*}{$\operatorname{Dev}(\%)$} & \multirow{2}{*}{$\mathrm{CPU}$} & \multicolumn{2}{|c|}{ Best Result } & \multicolumn{3}{|c|}{ Average Result } \\
\hline & & & & & $\mathrm{Z}$ & $\operatorname{Dev}(\%)$ & $\mathrm{Z}$ & $\operatorname{Dev}(\%)$ & $\mathrm{CPU}$ \\
\hline 6 & $56,364.35$ & $58,523.45$ & 3.6893 & $10,803.73$ & $56,364.35$ & 0.0000 & $56,511.75$ & 0.2608 & $3,605.96$ \\
\hline 7 & $55,480.55$ & $57,194.35$ & 2.9964 & $10,839.48$ & $55,480.55$ & 0.0000 & $55,880.96$ & 0.7165 & $3,602.21$ \\
\hline 8 & $55,779.00$ & $58,203.70$ & 4.1659 & $10,864.55$ & $55,779.00$ & 0.0000 & $55,974.63$ & 0.3495 & $3,602.46$ \\
\hline 9 & $55,344.30$ & $59,605.60$ & 7.1492 & $10,951.32$ & $55,344.30$ & 0.0000 & $55,671.59$ & 0.5879 & $3,603.02$ \\
\hline 10 & $55,214.95$ & $60,925.70$ & 9.3733 & $10,812.67$ & $55,214.95$ & 0.0000 & $56,104.26$ & 1.5851 & $3,605.15$ \\
\hline Average & & & 5.4748 & $10,854.35$ & & 0.0000 & & 0.7000 & $3,603.76$ \\
\hline
\end{tabular}




\section{Conclusion}

In this paper, mathematical models are proposed for generating an optimal layout for an installation port for an offshore wind farm. The objective functions of the models are to minimise the total transportation cost of the components within the port. The shape of the subareas that need to be located in the port is rectangular where each subarea has several possible dimensional options to choose from. Exact method and matheuristic approaches are proposed to solve the problem. In the matheuristic approaches, a hybridization of exact method and VNS technique is proposed to find a good solution in a reasonable computing time.

Two datasets are used for evaluating the performance of the proposed methods. The first dataset is randomly generated whereas the second dataset uses the area of Port Ardersier in Scotland as a case study. The computational experiments show that the exact method is able to attain optimality in almost all instances whereas the matheuristic methods perform well and run much faster than the exact method.

In future research, the proposed models can be treated as a criterion in the wider port choice model and OWF supply chain modelling. Offshore wind is an emerging industry in which the use of operational research methods such as those proposed in this paper are required in order to provide optimal decision support and hence an efficient logistics strategy. The use of operational research techniques for layout and port configuration problem in other emerging marine renewable technologies, such as tidal and wave power, is an interesting area for future research.

\section{Acknowledgments}

The research leading to these results has received funding from the European Union Seventh Framework Programme under the agreement SCP2-GA-2013-614020 (LEANWIND: Logistic Efficiencies And Naval architecture for Wind Installations with Novel Developments). 


\section{Appendix A}

Let $\rho_{a, a^{\prime}, s, s^{\prime}}=d_{a, a^{\prime}, s, s^{\prime}} \cdot \lambda_{a, a^{\prime}, s, s^{\prime}}$, so $\rho_{a, a^{\prime}, s, s^{\prime}}=\left(\left|\left(x_{a, s}+\frac{l_{a, s}}{2}\right)-\left(x_{a^{\prime}, s^{\prime}}+\frac{l_{a^{\prime}, s^{\prime}}}{2}\right)\right|+\left|\left(y_{a, s}+\frac{w_{a, s}}{2}\right)-\left(y_{a^{\prime}, s^{\prime}}+\frac{w_{a}, s^{\prime}}{2}\right)\right|\right) \cdot \lambda_{a, a^{\prime}, s, s^{\prime}}$

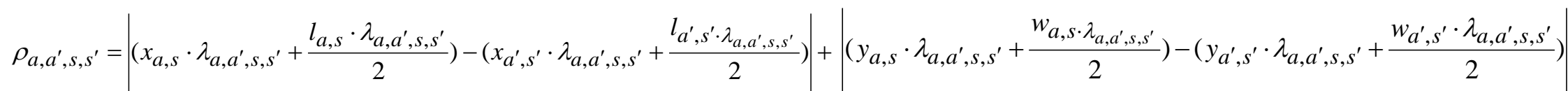

Let $\quad \hat{X}_{a, a^{\prime}, s, s^{\prime}}=x_{a, s} \cdot \lambda_{a, a^{\prime}, s, s^{\prime}}, \tilde{X}_{a, a^{\prime}, s, s^{\prime}}=x_{a^{\prime}, s^{\prime}} \cdot \lambda_{a, a^{\prime}, s, s^{\prime}}, \hat{Y}_{a, a^{\prime}, s, s^{\prime}}=y_{a, s} \cdot \lambda_{a, a^{\prime}, s, s^{\prime}}$, and $\tilde{Y}_{a, a^{\prime}, s, s^{\prime}}=y_{a^{\prime}, s^{\prime}} \cdot \lambda_{a, a^{\prime}, s, s^{\prime}} \cdot$ These new variables can be substituted into Equation (A1) which can be re-written as follows:

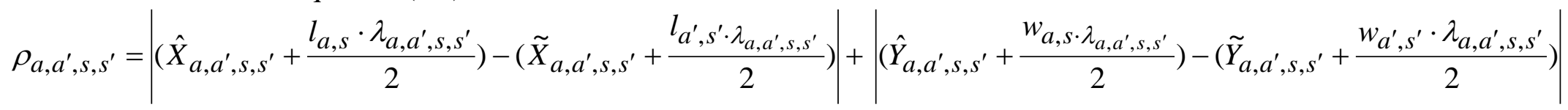

By substituting Equation A2 into the objective function (Equation 1), the model becomes a linear problem.

$Z^{c}=\sum_{q \in Q} \sum_{a \in A} \sum_{a^{\prime} \in A} \sum_{s \in S_{a}} \sum_{s^{\prime} \in S_{a^{\prime}}} \rho_{a, a^{\prime}, s, s^{\prime}} \cdot c_{q} \cdot m_{q, a, a^{\prime}}$

However, new constraints need to be added into the model as follows:
$\hat{X}_{a, a^{\prime}, s, s^{\prime}} \leq X_{\max } \cdot \lambda_{a, a^{\prime}, s, s^{\prime}}, \forall a, a^{\prime} \in A ; s \in S_{a} ; s^{\prime} \in S_{a^{\prime}}$
(A4)
$\hat{X}_{a, a^{\prime}, s, s^{\prime}} \geq X_{\min } \cdot \lambda_{a, a^{\prime}, s, s^{\prime}}, \forall a, a^{\prime} \in A ; s \in S_{a} ; s^{\prime} \in S_{a^{\prime}}$
$\hat{Y}_{a, a^{\prime}, s, s^{\prime}} \leq Y_{\max } \cdot \lambda_{a, a^{\prime}, s, s^{\prime}}, \forall a, a^{\prime} \in A ; s \in S_{a} ; s^{\prime} \in S_{a^{\prime}}$
$\hat{X}_{a, a^{\prime}, s, s^{\prime}} \leq x_{a, s}-X_{\min } \cdot\left(1-\lambda_{a, a^{\prime}, s, s^{\prime}}\right), \forall a, a^{\prime} \in A ; s \in S_{a} ; s^{\prime} \in S_{a^{\prime}}$
$\hat{Y}_{a, a^{\prime}, s, s^{\prime}} \geq Y_{\min } \cdot \lambda_{a, a^{\prime}, s, s^{\prime}}, \forall a, a^{\prime} \in A ; s \in S_{a} ; s^{\prime} \in S_{a^{\prime}}$
$\hat{X}_{a, a^{\prime}, s, s^{\prime}} \geq x_{a, s}-X_{\max } \cdot\left(1-\lambda_{a, a^{\prime}, s, s^{\prime}}\right), \forall a, a^{\prime} \in A ; s \in S_{a} ; s^{\prime} \in S_{a^{\prime}}$
(A6) $\quad \hat{Y}_{a, a^{\prime}, s, s^{\prime}} \leq y_{a, s}-Y_{\min } \cdot\left(1-\lambda_{a, a^{\prime}, s, s^{\prime}}\right), \forall a, a^{\prime} \in A ; s \in S_{a} ; s^{\prime} \in S_{a^{\prime}}$
$\tilde{X}_{a, a^{\prime}, s, s^{\prime}} \leq X_{\max } \cdot \lambda_{a, a^{\prime}, s, s^{\prime}}, \forall a, a^{\prime} \in A ; s \in S_{a} ; s^{\prime} \in S_{a^{\prime}}$
$\hat{Y}_{a, a^{\prime}, s, s^{\prime}} \geq y_{a, s}-Y_{\max } \cdot\left(1-\lambda_{a, a^{\prime}, s, s^{\prime}}\right), \forall a, a^{\prime} \in A ; s \in S_{a} ; s^{\prime} \in S_{a^{\prime}}$
$\tilde{X}_{a, a^{\prime}, s, s^{\prime}} \geq X_{\min } \cdot \lambda_{a, a^{\prime}, s, s^{\prime}}, \forall a, a^{\prime} \in A ; s \in S_{a} ; s^{\prime} \in S_{a^{\prime}}$
(A8) $\quad \tilde{Y}_{a, a^{\prime}, s, s^{\prime}} \leq Y_{\max } \cdot \lambda_{a, a^{\prime}, s, s^{\prime}}, \forall a, a^{\prime} \in A ; s \in S_{a} ; s^{\prime} \in S_{a^{\prime}}$
$\tilde{X}_{a, a^{\prime}, s, s^{\prime}} \leq x_{a^{\prime}, s^{\prime}}-X_{\min } \cdot\left(1-\lambda_{a, a^{\prime}, s, s^{\prime}}\right), \forall a, a^{\prime} \in A ; s \in S_{a} ; s^{\prime} \in S_{a^{\prime}}$
$\tilde{Y}_{a, a^{\prime}, s, s^{\prime}} \geq Y_{\min } \cdot \lambda_{a, a^{\prime}, s, s^{\prime}}, \forall a, a^{\prime} \in A ; s \in S_{a} ; s^{\prime} \in S_{a^{\prime}}$
$\tilde{X}_{a, a^{\prime}, s, s^{\prime}} \geq x_{a^{\prime}, s^{\prime}}-X_{\max } \cdot\left(1-\lambda_{a, a^{\prime}, s, s^{\prime}}\right), \forall a, a^{\prime} \in A ; s \in S_{a} ; s^{\prime} \in S_{a^{\prime}}$
$\tilde{Y}_{a, a^{\prime}, s, s^{\prime}} \leq y_{a^{\prime}, s^{\prime}}-Y_{\min } \cdot\left(1-\lambda_{a, a^{\prime}, s, s^{\prime}}\right), \forall a, a^{\prime} \in A ; s \in S_{a} ; s^{\prime} \in S_{a^{\prime}}$ (A18)

Where $X_{\min }=\min _{i}\left(x_{i}\right), Y_{\min }=\min _{i}\left(y_{i}\right), X_{\max }=\max _{i}\left(x_{i}\right), Y_{\max }=\max _{i}\left(y_{i}\right), i \in V$ with $V$ is a set of vertices $\left(x_{i}, y_{i}\right)$ that form the port area. 


\section{Appendix B}

Table B1. List of subareas with their possible rectangles (Instance 2a)

\begin{tabular}{clcccccccccc}
\hline \multirow{2}{*}{$\begin{array}{c}\text { Area } \\
\text { ID }\end{array}$} & Area Name & \multicolumn{2}{c}{ Dimension 1} & \multicolumn{2}{c}{ Dimension 2} & \multicolumn{2}{c}{ Dimension 3} & \multicolumn{3}{c}{ Dimension 4 } & \multicolumn{2}{c}{ Dimension 5 } \\
\cline { 2 - 12 } & & $l$ & $w$ & $l$ & $w$ & $l$ & $w$ & $l$ & $w$ & $l$ & $w$ \\
\hline A1 & Loading Area & 500.00 & 200.00 & 200.00 & 500.00 & 250.00 & 400.00 & 400.00 & 250.00 & 450.00 & 222.22 \\
A2 & Tower Stage & 350.00 & 144.00 & 144.00 & 350.00 & 150.00 & 336.00 & 336.00 & 150.00 & 140.00 & 360.00 \\
A3 & Nacelle Stage & 350.00 & 144.00 & 144.00 & 350.00 & 150.00 & 336.00 & 336.00 & 150.00 & 140.00 & 360.00 \\
A4 & Blade Stage & 400.00 & 600.00 & 600.00 & 400.00 & 500.00 & 480.00 & 480.00 & 500.00 & 375.00 & 640.00 \\
A5 & Tower Storage & 300.00 & 300.00 & 250.00 & 360.00 & 360.00 & 250.00 & 200.00 & 450.00 & 450.00 & 200.00 \\
A6 & Nacelle Storage & 300.00 & 48.00 & 48.00 & 300.00 & 60.00 & 240.00 & 240.00 & 60.00 & 250.00 & 57.60 \\
A7 & Blade Storage & 300.00 & 140.00 & 140.00 & 300.00 & 150.00 & 280.00 & 280.00 & 150.00 & 160.00 & 262.50 \\
A8 & Unloading Area & 500.00 & 150.00 & 468.75 & 160.00 & 535.71 & 140.00 & 517.24 & 145.00 & 483.87 & 155.00 \\
\hline
\end{tabular}

Table B2. List of subareas with their possible rectangles (Instance 2b)

\begin{tabular}{|c|c|c|c|c|c|c|c|c|c|c|c|c|c|c|c|c|c|c|c|c|c|}
\hline \multirow{2}{*}{$\begin{array}{l}\text { Area } \\
\text { ID }\end{array}$} & \multirow{2}{*}{ Area Name } & \multicolumn{2}{|c|}{ Dimension 1} & \multicolumn{2}{|c|}{ Dimension 2} & \multicolumn{2}{|c|}{ Dimension 3} & \multicolumn{2}{|c|}{ Dimension 4} & \multicolumn{2}{|c|}{ Dimension 5} & \multicolumn{2}{|c|}{ Dimension 6} & \multicolumn{2}{|c|}{ Dimension 7} & \multicolumn{2}{|c|}{ Dimension 8} & \multicolumn{2}{|c|}{ Dimension 9} & \multicolumn{2}{|c|}{ Dimension 10} \\
\hline & & $l$ & $w$ & $l$ & $w$ & $l$ & $w$ & $l$ & $w$ & $l$ & $w$ & $l$ & $W$ & $L$ & $w$ & $l$ & $w$ & $l$ & $w$ & $l$ & $w$ \\
\hline A1 & Loading Area & 375 & 200 & 200 & 375 & 250 & 300 & 300 & 250 & 240 & 312.5 & 312.5 & 240 & 235 & 319.5 & 319.15 & 235 & 260 & 288.5 & 288.5 & 260 \\
\hline A2 & Tower Stage & 262.5 & 144 & 144 & 262.5 & 135 & 280 & 280 & 135 & 140 & 270 & 270 & 140 & 150 & 252 & 252 & 150 & 168 & 225 & 225 & 168 \\
\hline A3 & Nacelle Stage & 262.5 & 144 & 144 & 262.5 & 135 & 280 & 280 & 135 & 140 & 270 & 270 & 140 & 150 & 252 & 252 & 150 & 168 & 225 & 225 & 168 \\
\hline A4 & Blade Stage & 400 & 200 & 200 & 400 & 250 & 320 & 320 & 250 & 210 & 281 & 281 & 210 & 230 & 248 & 248 & 230 & 240 & 233 & 233 & 240 \\
\hline A5 & Foundation Stage & 400 & 200 & 200 & 400 & 250 & 320 & 320 & 250 & 210 & 281 & 281 & 210 & 230 & 248 & 248 & 230 & 240 & 233 & 233 & 240 \\
\hline A6 & Transition Piece Sta. & 262.5 & 144 & 144 & 262.5 & 135 & 280 & 280 & 135 & 140 & 270 & 270 & 140 & 150 & 252 & 252 & 150 & 168 & 225 & 225 & 168 \\
\hline A7 & Tower Storage & 225 & 300 & 300 & 225 & 216 & 312.5 & 312.5 & 216 & 240 & 281.25 & 281.25 & 240 & 250 & 270 & 270 & 250 & 260 & 259 & 259 & 260 \\
\hline A8 & Nacelle Storage & 225 & 48 & 48 & 225 & 216 & 50 & 50 & 216 & 200 & 54 & 54 & 200 & 192 & 56.25 & 56.25 & 192 & 180 & 60 & 60 & 180 \\
\hline A9 & Blade Storage & 225 & 140 & 140 & 225 & 250 & 126 & 126 & 250 & 210 & 150 & 150 & 210 & 200 & 157.5 & 157.5 & 200 & 180 & 175 & 175 & 180 \\
\hline A10 & Foundation Storage & 225 & 140 & 140 & 225 & 250 & 126 & 126 & 250 & 210 & 150 & 150 & 210 & 200 & 157.5 & 157.5 & 200 & 180 & 175 & 175 & 180 \\
\hline A11 & Transition Piece Sto. & 225 & 48 & 48 & 225 & 216 & 50 & 50 & 216 & 200 & 54 & 54 & 200 & 192 & 56.25 & 56.25 & 192 & 180 & 60 & 60 & 180 \\
\hline $\mathrm{A} 12$ & Unloading Area & 375 & 150 & 401.8 & 140 & 387.9 & 145 & 362.9 & 155 & 362.9 & 160 & 396 & 142 & 390.5 & 144 & 365 & 154 & 370 & 152 & 360 & 156 \\
\hline
\end{tabular}




\section{References}

1. Askin, R.G., \& Standridge, C.R. (1993). Modeling and Analysis of Manufacturing System. New York: Wiley.

2. Alvarez-Valdes, R., Martinez, A. \& Tamarit, J.M. (2013). A branch \& bound algorithm for cutting and packing irregularly shaped pieces, 145 (2), International Journal of Production Economics, 463-477.

3. Balakrishnan, J., Cheng, C. H., Conway, D. G., \& Lau, C. M. (2003). A hybrid genetic algorithm for the dynamic plant layout problem. International Journal of Production Economics, 86(2), 107-120.

4. Brimberg, J., \& Mladenovic, N. (1996). A variable neighbourhood algorithm for solving the continuous location-allocation problem. Studies of Locational Analysis, 10, 1-12.

5. Castillo, I., Westerlund, J., Emet, S. \& Westerlund, T. (2005). Optimiztion of block layout design problems with unequal areas: A comparison of MILP and MINLP optimization methods. Computers and Chemical Engineering, 30(1), 54-69.

6. Chen, Y., Jiang, Y., Wahab, M.I.M., \& Long, X. (2015). The facility layout problem in non-rectangular logistics parks with split lines. Expert systems with applications. 42, 7768-7780.

7. Chernov, N., Stoyan, Y., Romanova, T. (2009). Mathematical model and efficient algorithms for objects packing problem. Computational Geometry: Theory and Applications, DOI: DOI: 10.1016/ j.comgeo.2009.12.003.

8. Chwif, L., Barretto, M. R. P., Moscato, L. A. (1998). Solution to the facility layout problem using simulated annealing. Computers in Industry, 36, 125-132

9. Das, S. K. (1993). A facility layout method for flexible manufacturing systems. International Journal of Production Research, 31(2), 279-297.

10. Degraeve, Z. \& Vandebroek, M. (1998). A Mixed Integer Programming Model for Solving a Layout Problem in the Fashion Industry. Management Science, 44, 301-310.

11. Dowsland, K.A., \& Dowsland, W.B. (1992). Packing Problems. European Journal of Operational Research, 56, 2-14.

12. Drira, A., Pierreval, H., \& Hajri-Gabouj, S. (2007). Facility layout problems: A survey. Annual Reviews in Control, 31, 255-267.

13. EWEA, 2015. The European offshore wind industry-key trends and statistics 2014. A report by the European Wind Energy Association. Available online at: http://www.ewea.org/fileadmin/files/library/publications/statistics/EWEA-EuropeanOffshore-Statistics-2014.pdf

14. Garey, M.R. \& Johnson, D.S. (1979). Computer and Intractability: A Guide to the Theory of NP-Completeness. San Francisco: W.H. Freeman.

15. Gonçalves, J. F., Mauricio G. C. (2015). A biased random-key genetic algorithm for the unequal area facility layout problem. European Journal of Operational Research 246, 86-107.

16. Kusiak, A., \& Heragu, S. S. (1987). The facility layout problem. European Journal of Operational Research, 29, 229-251.

17. Han, W., Bennell, J.A., Zhao, X.Z., \& Song, X. (2013). Construction heuristics for twodimensional irregular shape bin packing with guillotine constraints. European Journal of Operational Research, 230, 495-504.

18. Hansen, P., \& Mladenovic, N. (1997). Variable neighbourhood search for the $p$-median. Location Science, 5, 207-225. 
19. Hansen, P., \& Mladenovic, N. (2001). Variable neighbourhood search: Principles and applications. European Journal of Operational Research, 130, 449-467.

20. Hansen, P., Mladenovic, N., \& Perez, J.A.M. (2010). Variable neighbourhood search: methods and applications. Annals of Operations Research, 175, 367-407.

21. Heragu, S. S., \& Kusiak, A. (1991). Efficient models for the facility layout problem. European Journal of operational research, 53, 1-13.

22. Hopper, E., \& Turton, B.C.H. (2001). A Review of the Application of Meta-Heuristic Algorithms to 2D Strip Packing Problems. Artificial Intelligence Review, 16, 257-300.

23. Irawan, C.A., Jones , D., Ouelhadj, D. (2015). Bi-objective optimisation model for installation scheduling in offshore wind farms. Computers \& Operations Research, http://dx.doi.org/10.1016/j.cor.2015.09.010.

24. Kim, K.H., Park, Y-M., \& Jin, M-J. (2008). An optimal layout of container yards. $O R$ Spectrum, 30, 675-695.

25. Koopmans, T. C., Beckmann, M. (1957). Assignment Problems and the Location of Economic Activities. Econometrica, 25, 1, 53-76.

26. Kulturel-Konak, S., \& Konak, A. (2011). Unequal area flexible bay facility layout using ant colony optimisation. International Journal of Production Research, 49, 1877-1902.

27. Lee, G. C., \& Kim, Y. D. (2000). Algorithms for adjusting shapes of departments in block layouts on the gird-based plane. Omega, 28(1), 111-122.

28. Lee, K. Y., Roh, M. I.,\&Jeong, H. S. (2005). An improved genetic algorithm for multifloor facility layout problems having inner structure walls and passages. Computers \& Operations Research, 32(4), 879-899.

29. Liu, C.I., Jula, H., Vukadinovic, K., \& Ioannou, P. (2004). Automated guided vehicle system for two container yard layouts. Transportation Research Part C: Emerging Technologies, 12, 349-368.

30. Lodi, A., Martello, S., \& Vigo, D. (1999), Approximation algorithms for the oriented two-dimensional bin packing problem. European Journal of Operational Research, 112, $158-166$.

31. Lodi, A., Martello, S., \& Vigo, D. (2002a). Recent Advances on Two-Dimensional Bin Packing Problems. Discrete Applied Mathematics, 123, 379-396.

32. Lodi, A., Martello, S., \& Vigo, D. (2002b). Two-Dimensional Packing Problems: A Survey. European Journal of Operational Research, 141, 241-252.

33. Martens, J. (2004). Two genetic algorithms to solve a layout problem in the fashion industry. European Journal of Operational Research, 154, 304-332.

34. Martinez-Sykora, A., Alvarez-Valdez, R., Bennell, J.A., \& Tamarit, J.M. (2015). Constructive procedures to solve 2-dimensional bin packing problems with irregular pieces and guillotine cuts. Omega, 52, 15-32.

35. Matai, R. (2015). Solving multi-objective facility layout problem by modified simulated annealing. Applied Mathematics and Computation, 261, 302-311.

36. McKendall, A.R., Noble, J.S., \& Klein, C.M. (1999). Facility layout of irregular-shaped departments using a nested approach. International Journal of Production Research, 37, 2895-2914.

37. Meller, R. D., \& Gau, K. -Y. (1996). The facility layout problem: recent and emerging trends and perspectives. Journal of Manufacturing Systems, 15, 351-366.

38. Meller, R. D., Narayanan, V., Vance, P. H. (1999). Optimal facility layout design. Operations Research Letters, 23, 117-127.

39. Meng, G., Heragu, S. S., \& Zijm, H. (2004). Reconfigurable layout problem. International Journal of Production Research, 42(22), 4709-4729. 
40. Montreuil, B. (1990). A Modeling framework for integrating layout design and flow network design, Proc. Materials Handling Research Colloq., Hebron, KY, pp. 43 - 58.

41. Neghabi, H., Tari, F.G. (2016). A new concept of adjacency for concurrent consideration of economic and safety aspects in design of facility layout problems, Journal of Loss Prevention in the Process Industries, 40, 603-614.

42. Perveen, R., Kishor, N., \& Mohanty, S.R. (2014). Off-shore wind farm development: present status and challenges. Renewable and Sustainable Energy Reviews, 29, 780-792

43. Petering, M.E.H. (2008). Parallel versus perpendicular yard layouts for seaport container transshipment terminals: an extensive simulation analysis. In: Proceedings of the International Trade and Freight Transportation Conference, Ayia Napa, Cyprus.

44. Petering, M.E.H. (2009). Effect of block width and storage yard layout on marine container terminal performance. Transportation Research Part E: Logistics and Trasnportation Review, 45, 591-610.

45. Rashidi. H., \& Tsang. E.P.K., (2013). Novel constraints satisfaction models for optimization problems on container yard terminal. Applied Mathematical Modelling, 37, 3601-3634.

46. Ripon, K.S.N., Khan, K.G.K.N., Hovin, M., Torresen, J. (2013). Adaptive variable neighborhood search for solving multi-objective facility layout problems with unequal area facilities. Swarm and Evolutionary Computation, 8, 1-12.

47. Stoyan, Y., Terno, J., Scheithauer, G., Gil, N., \& Romanova, T. (2001). Phi-functions for primary 2D-objects. Studia Informatica Universalis, 2, 1-32.

48. Stoyan, Y., Scheithauer, G., Gil, N., \& Romanova, T. (2004). Ф-functions for complex 2D-objects. 4OR: Quarterly, J. Belgian, French and Italian Operations Research Soc, 2 , 69-84.

49. Scheithauer G., Stoyan Y., Gil N., \& Romanova T. (2003). Phi-functions for circular segments. Dresden: Prepr. Technische Univarsitat Dresden.

50. Scheithauer, G., Stoyan, Y., Ye, T., \& Romanova, T. (2005). Mathematical modelling of interactions of primary geometric 3D objects. Cybernet Systems Anal, 41, 332-342.

51. Sherali, H. D., Fraticelli, B. M. P., Meller, R. D. (2003). Enhanced model formulations for optimal facility layout. Operations Research, 51, 4, 629-644.

52. Tam, K.Y. , Li, S.G. (1991). A hierarchical approach to the facility layout problem, Int. J. Prod. Res., 29, 1, 165-184.

53. Thomson, K.E. (2012). Offshore wind a comprehensive guide to successful offshore wind farm installation. Massachusetts: Elsevier.

54. Tompkins, J., White, J., Bozer, Y., Frazelle, E., Tanchoco, J., \& Trevino, J. (1996). Facilities planning. New York: Wiley.

55. Valerio de Carvalho, J.M. (1999). Exact Solution of Bin-Packing Problems using Column Generation and Branch and Bound. Annals of Operations Research, 86, 629-659.

56. Vanderbeck, F. (1999). Computational Study of a Column Generation Algorithm for Bin Packing and Cutting Stock Problems. Mathematical Programming, 86, 565-594.

57. Wäscher, G., Haußner, H., \& Schumann, H. (2007). An improved typology of cutting and packing problems. European Journal of Operational Research, 183, 1109-1130.

58. Wiese, J., Suhl, L., Kliewer, N. (2010). Mathematical models and solution methods for optimal container terminal yard layouts. OR Spectrum, 32, 427-452.

59. Zhang, M., Savas,S., Batta, R., Nagi, R. (2009). Facility placement with sub-aisle design in an existing layout, European Journal of Operational Research, 197, 1, 154-165. 